Homology, Homotopy and Applications, vol.9(1), 2007, pp.107-138

\title{
SIMPLICIAL MODEL CATEGORY STRUCTURES ON THE CATEGORY OF CHAIN FUNCTORS
}

\author{
FRIEDRICH W. BAUER AND TAMAR DATUASHVILI
}

(communicated by Jim Stasheff)

\begin{abstract}
The model structure on the category of chain functors $\mathfrak{C h}$, developed in [4], has the main features of a simplicial model category structure, taking into account the lack of arbitrary (co-)limits in $\mathfrak{C h}$. After an appropriate tensor and cotensor structure in $\mathfrak{C h}$ is established $(\S 1, \S 3)$, Quillen's axiom SM7 is verified in $\S 5$ and $\S 6$. Moreover, it turns out that in the definition of a simplicial model structure, the category of simplicial sets can be replaced by the category of simplicial spectra endowing $\mathfrak{C h}$ with the structure of an approximate simplicial stable model structure (= approximate ss-model structure) ( $\S 7)$. In $\S 8$ the model structure on $\mathfrak{C} h$ is shown to be proper.
\end{abstract}

\section{Introduction}

To motivate chain functors as tools for the calculation of generalized homology theories by means of chain complexes (as used for ordinary homology theories) we refer to [1] and, in a very condensed form, to $\S 10$ below. The objective of the present paper is to investigate to what extent the model structure on the category $\mathfrak{C h}$ of chain functors, introduced in [4], becomes a simplicial one (admitting a tensor and a cotensor product, satisfying different formulations of Quillen's axiom SM7). Hence we are, as in $[3,4]$, dealing with properties of the category $\mathfrak{C h}$.

The definition of a chain functor is recorded in [4], $\S 7$ and, in order to make the present paper independent, in $\S 10$. We refer to this treatment whenever special properties of a chain functor are needed. This happens only in connection with the verification of Proposition 1.1, hence almost exclusively in $\S 9$, where a detailed proof is presented that the tensor product between a chain functor and a free abelian chain complex has the structure of a chain functor. Moreover, for the reader's convenience, we have included in $\S 11$ a short record of the original definition of a simplicial model category (including axioms SM6 and SM7 in the sense of D. Quillen [11]) as well as modifications of axiom SM6 that lead to what we call an approximate simplicial model structure.

Received October 29, 2005, revised June 4, 2006; published on January 11, 2007. 2000 Mathematics Subject Classification: 18G35, 55U15, 55U40, 55U35.

Key words and phrases: Chain functor, simplicial spectrum, fibration, cofibration.

Copyright (C) 2007, International Press. Permission to copy for private use granted. 
Since the structure we offer is not a plain simplicial model structure in the sense of Quillen, we explain in each case what specific properties are lacking or need changing. A typical example is the associativity of the tensor product ( $\$ 2(3)$, which is only available up to weak equivalences) and the existence of function objects in $\S 3$. In $\S 1$ we present the definition of a tensor product $\boldsymbol{A}_{*} \otimes K$, for $\boldsymbol{A}_{*} \in \mathfrak{C h}$ and $K$ in the category $\mathfrak{S}$ of simplicial sets, which is associative up to weak equivalence (more precisely, a so-called Eilenberg-Zilber mapping, see Lemma 2.7). The detailed proof of the existence of $\boldsymbol{A}_{*} \otimes K$, for $\boldsymbol{A}_{*} \in \mathfrak{C h}, K \in \mathfrak{S}$ (Proposition 1.1) is relegated to $\S 9$.

Since $\mathfrak{C h}$ does not contain arbitrary (co-)limits, the definition of $\boldsymbol{A}_{*}^{K}$ (the cotensor product) causes problems, which are settled in $\S 3$. In $\S 2$ we verify that $\boldsymbol{A}_{*} \otimes K$ behaves well for cofibrations. The function complex $\operatorname{Hom}\left(\boldsymbol{A}_{*}, \boldsymbol{B}_{*}\right) \in \mathfrak{S}$ for $\boldsymbol{A}_{*}, \boldsymbol{B}_{*} \in \mathfrak{C h}$ is introduced in $\S 4$ in the usual way, where the basic properties of this simplicial set (which is always a Kan set, cf. §4.1) are verified. So the expected behavior is obtained under (co-)fibrations, as is adjointness with the tensor product (Theorem 4.2). All of our constructions are presented in such a way as to be able to immediately transfer everything in $\S 7$ to simplicial spectra instead of simplicial sets.

The following sections are devoted to a verification of Quillen's axiom SM7 in the formulation for tensor products ( $\S 5$, Theorem 5.3) and for the complex Hom in $\S 6$, Theorem 6.2 (cf. [11], Ch. II, $\S 2$, Definition 2).

These goals can only be accomplished by application of the results in [3] concerning the existence of specific (co-)limits in $\mathfrak{C h}$.

As we pointed out above, in $\S 7$ we investigate the ss-model category properties (Definition 7.1) of $\mathfrak{C h}$, where the role of the category of simplicial sets is played by simplicial spectra. Consequently we now treat approximate ss-model structures. So Hom now appears as a simplicial spectrum. All results of $\S 1-\S 6$ are formulated in such a way that this can be immediately achieved.

$\S 8$ is devoted to the (left and right) properness of $\mathfrak{C h}$. For applications and more details on this concept, we refer to [7].

\section{Acknowledgements}

The second author is grateful for the financial support granted by DFG which facilitated the cooperation between the authors and therefore the present work.

\section{1. $\mathfrak{C h}$ as tensored category}

In this and the following six sections, we establish for the category $\mathfrak{C} h$ the expected part of a simplicial model structure (cf. §11). In the present section we start with the property that there is a tensor product between objects of $\mathfrak{C h}$ and objects of $\mathfrak{S}$ (cf. Definition 11.1).

We denote the category of complexes of free abelian groups by $\mathfrak{c h}$, i.e. a $C_{*} \in \mathfrak{c h}$ is a family of free abelian groups $C_{n}$, with boundary morphisms $d_{n}=d: C_{n} \longrightarrow$ $C_{n-1}, d_{n-1} d_{n}=0, n \in \mathbb{Z}$. Let $K \in \mathfrak{S}$ be a simplicial set. Then we have a free 
abelian group complex $C_{*}(K)$ associated with $K$, that is $C_{n}(K)$ is the free abelian group generated by the $n$-simplexes of $K$. An element $c \in C_{n}(K)$ is therefore a finite sum of the form

$$
\sum m_{i} \sigma_{i}^{n}, \sigma_{i}^{n} \in K_{n}, m_{i} \in \mathbb{Z}
$$

The boundary is defined by

$$
d \sigma^{n}=\sum_{i=0}^{n}(-1)^{i} \partial_{i}^{n} \sigma^{n} .
$$

We have a functor $C_{*}: \mathfrak{S} \longrightarrow \mathfrak{c h}$, and by an abuse of notation write $\boldsymbol{A}_{*} \otimes K$ instead of $\boldsymbol{A}_{*} \otimes C_{*}(K)$. Later, in $\S 7$, we will use the category $\mathfrak{S p}$ of simplicial spectra instead of $\mathfrak{S}$.

Let $\boldsymbol{A}_{*} \in \mathfrak{C h}$ be a chain functor, defined on a category $\mathfrak{K}$ (cf. $\S 10$ for more details), $K_{*} \in \mathfrak{c h}$. We then define a chain functor $\boldsymbol{B}_{*}=\boldsymbol{A}_{*} \otimes K_{*}$ in the following way: Let $(X, U) \in \mathfrak{K}$ be given. Then

$$
\begin{gathered}
B_{n}(X, U)=\left(A_{*}(X, U) \otimes K_{*}\right)_{n}=\bigoplus_{p+q=n} A_{p}(X, U) \otimes K_{q}, \\
d_{n}\left(a_{p} \otimes b_{q}\right)=d_{p} a_{p} \otimes k_{q}+(-1)^{p} a_{p} \otimes d_{q} k_{q} .
\end{gathered}
$$

This inherits the structure of a functor from $\boldsymbol{A}_{*}$ as follows: To each $f:(X, U) \longrightarrow$ $(Y, V)$ we have a $f_{\#} \otimes 1_{K_{*}}:\left(\boldsymbol{A}_{*} \otimes K_{*}\right)(X, U) \longrightarrow\left(\boldsymbol{A}_{*} \otimes K_{*}\right)(Y, V)$. We achieve the ingredients of a chain functor:

$$
\begin{gathered}
B_{n}^{\prime}(X, U)=\left(A_{n}^{\prime}(X, U) \otimes K_{*}\right)_{n} \\
\varphi_{B}=\varphi \otimes 1_{K_{*}}: A_{*}^{\prime}(X, U) \otimes K_{*} \longrightarrow A_{*}(X) \otimes K_{*} \\
\kappa_{B}=\kappa \otimes 1_{K_{*}}: A_{*}(X) \otimes K_{*} \longrightarrow A_{*}^{\prime} \otimes K_{*} .
\end{gathered}
$$

The inclusion $j: X \subset(X, U)$ induces a homomorphism

$$
j_{\#}^{B}: A_{*}(X) \otimes K_{*} \longrightarrow A_{*}(X, U) \otimes K_{*} .
$$

Tensoring with $1_{K_{*}}$ yields chain homotopies

$$
\varphi_{B} \kappa_{B} \simeq 1, j_{\#}^{B} \varphi_{B} \simeq l_{B},
$$

where $l_{B}: A_{*}^{\prime}(X, U) \otimes K_{*} \subset A_{*}(X, U) \otimes K_{*}$ is an inclusion (which exists, since $K_{*}$ is supposed to be free).

There is a natural inclusion

$$
i_{B}^{\prime}=i^{\prime} \otimes 1_{K_{*}}: A_{*}(U) \otimes K_{*} \subset A_{*}^{\prime}(X, U) \otimes K_{*},
$$

satisfying

$$
\kappa_{B} i_{\#}^{B}=i_{B}^{\prime}
$$

Suppose $f:(X, U) \subset(Y, B)$ is an inclusion. Then $A_{*}(f)$ is an inclusion (by CH2) and (because $K_{*}$ is free) so is $\left(A_{*} \otimes K_{*}\right)(f)$. Since $A_{*}(X, X)$ is acyclic, so is $\left(A_{*} \otimes\right.$ $\left.K_{*}\right)(X, X)$. This confirms that properties $\mathrm{CH} 1, \mathrm{CH} 2$ of a chain functor (cf. $\S 10$, [4], $\S 9$ or [3], §7) are satisfied for $\boldsymbol{B}_{*}=\boldsymbol{A}_{*} \otimes K_{*}$. 
Proposition 1.1. These constructions yield a functor

$$
-\otimes-: \mathfrak{C h} \times \mathfrak{c h} \longrightarrow \mathfrak{C h},
$$

Hence a functor

$$
-\otimes-: \mathfrak{C h} \times \mathfrak{S} \longrightarrow \mathfrak{C h} .
$$

A detailed proof is deferred to $\S 9$.

Let $K \in \mathfrak{S}$ be any simplicial set. Then instead of $C_{*}(K)$ we can employ the free sub-chain complex $\iota_{K}: \tilde{C}_{*}(K) \subset C_{*}(K)$, which is generated in each dimension by the non-degenerate simplexes of $K$. Together with this inclusion we have a retraction $\rho_{K}: C_{*}(K) \longrightarrow \tilde{C}_{*}(K)$ which maps all generators coming from degenerate simplexes to zero. It is obvious that

$$
\rho_{K} \iota_{K}=1, \iota_{K} \rho_{K} \simeq 1
$$

In particular, the cylinder object $\boldsymbol{A}_{*} \times I=\boldsymbol{A}_{*} \times \Delta^{1}$ introduced in [4], $\S 1$ is not isomorphic to $\boldsymbol{A}_{*} \otimes I=\boldsymbol{A} \otimes C_{*}(I)$ but to $\boldsymbol{A}_{*} \otimes \tilde{C}_{*}(I)$.

Two mappings $f_{0}, f_{1}: \boldsymbol{A}_{*} \longrightarrow \boldsymbol{B}_{*}$ are homotopic whenever there exists a homotopy $H: \boldsymbol{A}_{*} \times I \longrightarrow \boldsymbol{B}_{*}, H i_{k}=f_{k}, k=0,1$. However the previous considerations imply that we can equally well express a homotopy by a mapping $\hat{H}: \boldsymbol{A}_{*} \otimes I \longrightarrow$ $\boldsymbol{B}_{*}, \hat{H}=H \rho_{K}$.

We summarize:

Lemma 1.2. 1) To each $K \in \mathfrak{S}$ there is associated a natural inclusion $\iota_{K}: \tilde{C}_{*}(K)$ $\subset C_{*}(K)$ as well as a natural retraction $\rho_{K}: C_{*}(K) \longrightarrow \tilde{C}_{*}(K)$ such that (1) holds.

2) Two mappings $f_{0}, f_{1}: \boldsymbol{A}_{*} \longrightarrow \boldsymbol{B}_{*}$ in $\mathfrak{C h}$ are chain homotopic whenever there exists an $H: \boldsymbol{A}_{*} \otimes \Delta^{1} \longrightarrow \boldsymbol{B}_{*}$ such that

$$
H\left(1_{\boldsymbol{A}_{*}} \otimes \partial_{k}\right)=f_{k}, \partial_{k}: \Delta^{0} \subset \Delta^{1}, k=0,1 .
$$

\section{Tensor products and cofibrations}

Lemma 2.1. Let $q: \boldsymbol{A}_{*} \subset \boldsymbol{B}_{*}$ be a cofibration in $\mathfrak{C h}$ and $K_{*} \in \mathfrak{c h}$. Then $q \otimes 1: \boldsymbol{A}_{*} \otimes$ $K_{*} \longrightarrow \boldsymbol{B}_{*} \otimes K_{*}$ (hence $q \otimes 1: \boldsymbol{A}_{*} \otimes K \longrightarrow \boldsymbol{B}_{*} \otimes K, K \in \mathfrak{S}$ ) is a cofibration in $\mathfrak{C h}$.

Proof. According to [3] , Lemma 1.2 we have natural, levelwise splittings $B_{n}(X, U) \approx$ $A_{n}(X, U) \oplus \overline{A_{n}(X, U)}$ (respecting $l$ and $i^{\prime}$ ) for each $n \in \mathbb{Z}$. Thus we obtain a splitting

$$
B_{p}(X, U) \otimes K_{q} \approx\left(A_{p}(X, U) \otimes K_{q}\right) \oplus\left(\overline{A_{p}(X, U)} \otimes K_{q}\right)
$$

hence a splitting

$$
\left(B_{*} \otimes K_{*}\right)_{n}(X, U) \approx\left(\left(A_{*} \otimes K_{*}\right) \oplus \overline{\left(A_{*} \otimes K_{*}\right)}\right)_{n}(X, U)
$$

of the same kind.

Lemma 2.2. Let $\alpha: \boldsymbol{A}_{*} \longrightarrow \boldsymbol{B}_{*}$ be a weak equivalence and $K_{*} \in \mathfrak{c h}$. Then $\alpha \otimes$ 1: $\boldsymbol{A}_{*} \otimes K_{*} \longrightarrow \boldsymbol{B}_{*} \otimes K_{*}$ (hence also $\alpha \otimes 1: \boldsymbol{A}_{*} \otimes K \longrightarrow \boldsymbol{B}_{*} \otimes K, K \in \mathfrak{S}$ ) is a weak equivalence in $\mathfrak{C h}$. 
Proof. By definition, $\alpha$ is a homotopy equivalence. Hence we have a homotopy inverse $\beta: \boldsymbol{B}_{*} \longrightarrow \boldsymbol{A}_{*}$ and homotopies $D: \beta \alpha \simeq 1, H: \alpha \beta \simeq 1$. Tensoring these homotopies gives the result.

Lemma 2.3. 1) Let $\alpha: K_{*} \longrightarrow L_{*}$ be a chain homotopy equivalence in $\mathfrak{c h}$. Then

$$
1 \otimes \alpha: \boldsymbol{A}_{*} \otimes K_{*} \longrightarrow \boldsymbol{A}_{*} \otimes L_{*}
$$

is a weak equivalence (hence a homotopy equivalence) in $\mathfrak{C h}$ for any $\boldsymbol{A}_{*} \in \mathfrak{C h}$.

2) Let $f: K \longrightarrow L$ be a weak equivalence in $\mathfrak{S}$. Then $1 \otimes f: \boldsymbol{A}_{*} \otimes K \longrightarrow \boldsymbol{A}_{*} \otimes L$ is a weak equivalence in $\mathfrak{C h}$.

Proof. 1) We again tensor the relevant homotopies.

2) Observe that the functor $C_{*}: \mathfrak{S} \longrightarrow \mathfrak{c h}$ factors over the category $\mathfrak{S}_{a b}$ of free abelian simplicial groups. Hence the weak equivalence $f$ induces a weak equivalence of abelian simplicial groups $C_{*}(f): C_{*}(K) \longrightarrow C_{*}(L)$ (cf. [7]). However such a weak equivalence is always a homotopy equivalence (cf. [6], [7]), inducing a homotopy equivalence in $\mathfrak{c h}$. Now the result follows from 1 ).

Lemma 2.4. Let $q: K \subset L$ be a cofibration in $\mathfrak{S}$. Then

$$
1 \otimes q: \boldsymbol{A}_{*} \otimes K \longrightarrow \boldsymbol{A}_{*} \otimes L
$$

is a cofibration in $\mathfrak{C h}$ for any $\boldsymbol{A}_{*} \in \mathfrak{C h}$.

Proof. A cofibration in $\mathfrak{S}$ is simply an inclusion. Hence we accomplish a splitting

$$
\left(A_{*} \otimes L\right)_{n}(X, U) \approx\left(A_{*} \otimes K\right)_{n}(X, U) \oplus B_{n}(X, U)
$$

where $B_{*}(X, U)=A_{*}(X, U) \otimes M_{*}$ and $M_{*}$ is the free abelian graded group generated by all simplexes in $L \backslash K$. This splitting has all required properties.

We summarize:

Proposition 2.5. 1) Suppose $\boldsymbol{A}_{*} \in \mathfrak{C h}$ and $j: K \subset L$ is a (trivial) cofibration in S. Then $1 \otimes j: \boldsymbol{A}_{*} \otimes K \longrightarrow \boldsymbol{A}_{*} \otimes L$ is a (trivial) cofibration in $\mathfrak{C h}$.

2) Let $q: \boldsymbol{A}_{*} \subset \boldsymbol{B}_{*}$ be a (trivial) cofibration in $\mathfrak{C h}$ and $K \in \mathfrak{S}$. Then $q \otimes K$ is a (trivial) cofibration in $\mathfrak{C h}$.

Lemma 2.6. 1) The pushout of a (trivial) cofibration in $\mathfrak{C h}$ is a (trivial) cofibration.

2) The pullback of a (trivial) fibration in $\mathfrak{C h}$ is a (trivial) fibration. 
Proof. 1) Let

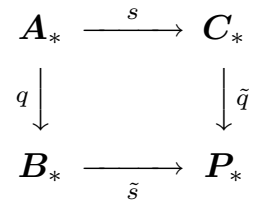

be a pushout in $\mathfrak{C h}, q$ a cofibration. Suppose

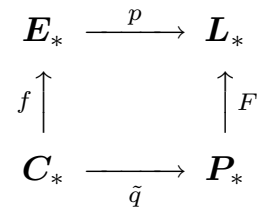

is a commutative square, with $p$ being a trivial fibration. Then a well-known argument displays a diagonal $\bar{F}: \boldsymbol{P}_{*} \longrightarrow \boldsymbol{E}_{*}$ rendering the square (2) commutative. The case of a trivial cofibration is settled by letting $p$ be any fibration.

2) This is entirely dual to 1).

We will return to a proof of a part of Lemma 2.6 in $\S 8.4$, using a slightly different terminology.

It is well-known that there are natural Eilenberg-Zilber maps (EZ-maps) [5], $\tau: C_{*}(K) \otimes C_{*}(L) \longrightarrow C_{*}(K \times L), K, L \in \mathfrak{S}$, which are homotopy equivalences and which, due to Lemma 2.3 , induce in all variables the natural homotopy equivalences

$$
\left(\boldsymbol{A}_{*} \otimes K\right) \otimes L \simeq \boldsymbol{A}_{*} \otimes(K \times L), \boldsymbol{A}_{*} \in \mathfrak{C h} .
$$

Moreover these EZ-maps have pleasant associativity properties: The AlexanderWhitney map ([5], Ch. VI 12.26) is strictly associative, yielding a strictly commutative diagram

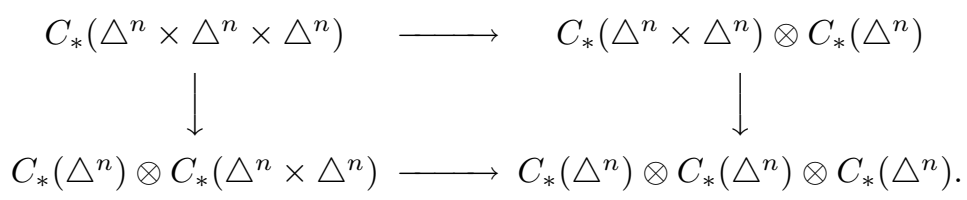

This is our form of associativity of the tensor product which will be needed in the proof of Proposition 4.5.

If $*=\Delta^{0} \in \mathfrak{S}$, we have a natural isomorphism

$$
\boldsymbol{A}_{*} \otimes \tilde{C}_{*}(*) \approx \boldsymbol{A}_{*}
$$

hence, because of Lemma 1.2 and Lemma 2.3 (1), a natural weak equivalence in $\mathfrak{C h}$

$$
\boldsymbol{A}_{*} \otimes * \simeq \boldsymbol{A}_{*} .
$$

Lemma 2.7. For any $K, L \in \mathfrak{S}$ and $\boldsymbol{A}_{*} \in \mathfrak{C h}$ we have a natural (with respect to all variables) weak equivalence (3) in $\mathfrak{C h}$, which is induced by the natural EZ-map. The point $* \in \mathfrak{S}$ serves as an identity for our tensor product, in the sense of (5). 
We can express (up to homotopy) the suspension $\Sigma \boldsymbol{A}_{*}$ of an object $\boldsymbol{A}_{*} \in \mathfrak{C h}$ by tensoring $\boldsymbol{A}_{*}$ with a sphere $S^{1}$ (i.e. a simplicial set having one non-degenerate simplex $\sigma^{1} \in\left(S^{1}\right)_{1}$ and one simplex $* \in\left(S^{1}\right)_{0}$; all other simplexes are degenerate).

In [3], $\S 1(1.7)$ as well as in [4], $\S 8$, we defined the suspension $\Sigma \boldsymbol{A}_{*}$ as the cokernel of the mapping

$$
\boldsymbol{A}_{*} \oplus \boldsymbol{A}_{*} \stackrel{i_{0} \oplus i_{1}}{\longrightarrow} \boldsymbol{A}_{*} \times I \longrightarrow \Sigma \boldsymbol{A}_{*} .
$$

Since $\boldsymbol{A}_{*} \times I=\boldsymbol{A}_{*} \otimes \tilde{C}_{*}(I)$, we obtain a homotopy equivalence between $\Sigma \boldsymbol{A}_{*}$ and $\tilde{\Sigma} \boldsymbol{A}_{*}=\boldsymbol{A}_{*} \otimes S^{1}$. The isomorphism between $A_{*} \otimes \tilde{C}_{*}\left(S^{1}\right)$ and the shift suspension $\bar{\Sigma} \boldsymbol{A}_{*}$ (cf. [3], $\S 1$ or $[4], \S 8$ ) is obvious.

Lemma 2.8. To any $\boldsymbol{A}_{*} \in \mathfrak{C h}$ there exists a natural weak equivalence (hence a homotopy equivalence)

$$
\tilde{\Sigma} \boldsymbol{A}_{*}=\boldsymbol{A}_{*} \otimes S^{1} \simeq \Sigma \boldsymbol{A}_{*} .
$$

\section{Function objects $\boldsymbol{A}_{*}^{K}$}

Let $\boldsymbol{A}_{*} \in \mathfrak{C h}, K_{*} \in \mathfrak{c h}, K_{*}=C_{*}(K), K \in \mathfrak{S}$. Then we can, analogous to $\boldsymbol{A}_{*} \otimes$ $K_{*}$, construct $\boldsymbol{A}_{*}^{K_{*}}$ ([5], Ch. II 1.9):

$$
\begin{gathered}
\left(A_{*}^{K_{*}}\right)_{n}(X, U)=\prod_{p \in \mathbb{Z}} \operatorname{Hom}\left(K_{p}, A_{n+p}(X, U)\right), \\
d\left\{f_{p}\right\}=\left\{d f_{p}-(-1)^{n} f_{p-1} d\right\}_{p \in \mathbb{Z} .}
\end{gathered}
$$

Here we understand by $K_{p}$, by an abuse of notation, the free abelian group generated by the $p$-simplexes of $K$. We set

$$
\left(A_{*}^{K_{*}}\right)_{n}^{\prime}(X, U)=\prod_{p \in \mathbb{Z}} \operatorname{Hom}\left(K_{p}, A_{n+p}^{\prime}(X, U)\right)
$$

and obtain the other ingredients of a chain functor, such as $\varphi, \kappa$ and the related chain homotopies as inherited from $\boldsymbol{A}_{*}$. This construction satisfies all properties of a chain functor, with one exception: $\left(A_{*}^{K_{*}}\right)_{n}(X, U)$ is not necessarily free. To this end we need the category $\mathfrak{C h h}$, with objects satisfying all properties of a chain functor $\mathrm{CH} 1-\mathrm{CH} 6$ but not $\mathrm{CH} 7$ (cf. [3], $\S 7$ or [4], $\S 9$ ). Thus we have a full inclusion of categories $\mathfrak{C h} \subset \mathfrak{C h h}$. We call an object of this larger category a chain complex functor. The fact that $\boldsymbol{A}_{*}^{K_{*}} \in \mathfrak{C h h}$ will be treated in $\S 9$ (Proposition 9.3). Moreover we know that $\boldsymbol{A}_{*} \otimes K_{*} \in \mathfrak{C h}$ for $\boldsymbol{A}_{*} \in \mathfrak{C h}$ (cf. Proposition 1.1), and the analogous assertion holds for $\boldsymbol{A}_{*} \in \mathfrak{C h h}$ (Corollary 9.2).

The next theorem provides us with a functor

$$
\Phi: \mathfrak{C h h} \longrightarrow \mathfrak{C h}
$$

exhibiting some pleasant properties.

Theorem 3.1. There exists a functor $\Phi$, as well as a natural transformation $\lambda_{\boldsymbol{A}_{*}}=$ $\lambda: \boldsymbol{L}_{*}=\Phi\left(\boldsymbol{A}_{*}\right) \longrightarrow \boldsymbol{A}_{*}, \boldsymbol{A}_{*} \in \mathfrak{C h h}$ (suppressing the inclusion $\mathfrak{C h} \subset \mathfrak{C h}$ from our notation), compatible with $l, i^{\prime}, \varphi, \kappa$, inducing an isomorphism of homology, such that: 
Homology, Homotopy and Applications, vol. 9(1), 2007

(L1) All $L_{*}(X, U)$ have a natural basis $\boldsymbol{b}$ in all dimensions.

(L2) $b \in \boldsymbol{b} \Rightarrow d b \in \boldsymbol{b} ; b \in \boldsymbol{b} \Rightarrow i^{\prime}(b) \in \boldsymbol{b} ; l(b) \in \boldsymbol{b}$ whenever this is defined.

(L3) To each $\zeta \in H_{*}\left(A_{*}(X, U)\right)$ there exists a cycle $z \in \zeta, z \in \boldsymbol{b}$.

The naturality of $\lambda$ of course means that the following square commutes:

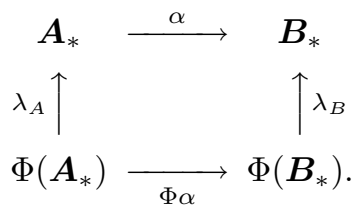

Proof. This is Lemma 9.1 in [4]. However, since we need one detail of the construction of $\Phi$, we briefly indicate the proof. The free generators of $\left(\Phi\left(\boldsymbol{A}_{*}\right)_{n}(X, U)\right)$ are 1) the elements $\bar{a}$ which are in 1-1 correspondence to the elements $a \neq 0$ in $A_{n}(X, U)$, and 2) elements of the cone over some subcomplex $M_{n}(X, U)$ which is generated by elements of the form

$$
\sum m_{i} \bar{a}_{i}-\overline{\sum m_{i} a_{i}}, m_{i} \in \mathbb{Z}
$$

The mapping $\lambda$ is defined by $\lambda(\bar{a})=a, \lambda(\sigma)=0, \sigma \in$ cone $M_{*}$.

Lemma 3.2. Let $\boldsymbol{A}_{*} \in \mathfrak{C h}, \boldsymbol{C}_{*} \in \mathfrak{C h h}, f \in \mathfrak{C h h}\left(\boldsymbol{A}_{*}, \boldsymbol{C}_{*}\right)$ be given. Then there exists an $\hat{f} \in \mathfrak{C h}\left(\boldsymbol{A}_{*}, \Phi\left(\boldsymbol{C}_{*}\right)\right)$ in a canonical way such that $\lambda \hat{f}=f$.

Proof. There exists a canonical basis in $\Phi\left(\boldsymbol{C}_{*}\right)$ which, according to the construction of $\Phi\left(\boldsymbol{C}_{*}\right)$, contains all elements of the form $\bar{a}, a \neq 0$ in $C_{*}(X, U)$ (i.e., all non-zero elements are sent to basis elements in $\left.\Phi\left(C_{*}\right)_{*}(X, U)\right)$. On the other hand we have a canonical basis $\boldsymbol{b}\left(\boldsymbol{A}_{*}\right)$ in $\boldsymbol{A}_{*}$. Let $b \in \boldsymbol{b}\left(\boldsymbol{A}_{*}\right) \cap A_{*}(X, U)$. Then we define

$$
\hat{f}(b)=\overline{f(b)} \in \boldsymbol{b}\left(\Phi\left(\boldsymbol{A}_{*}\right)\right) .
$$

This defines a morphism $\hat{f} \in \mathfrak{C h}\left(\boldsymbol{A}_{*}, \Phi\left(\boldsymbol{C}_{*}\right)\right)$, satisfying

$$
\lambda \hat{f}(a)=\overline{\hat{f}(a)}=f(a), a \in\left(\boldsymbol{A}_{*}\right) .
$$

We call a morphism $\tilde{p} \in \mathfrak{C h} \mathfrak{h}\left(\boldsymbol{E}_{*}, \boldsymbol{B}_{*}\right)$ a fibration whenever we have a levelwise natural section of $\bar{p}$ inducing an isomorphism

$$
E_{n}(X, U) \approx B_{n}(X, U) \oplus \overline{B_{n}(X, U)}
$$

which is compatible with $l$ and $i^{\prime}$. This definition is analogous to that in $\mathfrak{C h}$ (cf. $[3], 5.2)$.

Corollary 3.3. Let $\tilde{p}$ be a fibration in $\mathfrak{C h h}$. Then $\Phi(\tilde{p})=p \in \mathfrak{C h}\left(\Phi\left(\boldsymbol{E}_{*}\right), \Phi\left(\boldsymbol{B}_{*}\right)\right)$ is a fibration in $\mathfrak{C h}$.

Proof. The splitting (3) induces a splitting of $\left(\Phi\left(E_{*}\right)\right)_{n}(X, U)$. 
Suppose $K \in \mathfrak{S}$ is a simplicial set, $\boldsymbol{A}_{*} \in \mathfrak{C h}$. Then we denote $\boldsymbol{A}_{*}^{C_{*}(K)}$ by $\boldsymbol{A}_{*}^{K}$. It turns out (Proposition 9.3) that this defines a functor

$$
\mathfrak{C h} \mathfrak{h} \times \mathfrak{S}^{o p} \longrightarrow \mathfrak{C h} \mathfrak{h} .
$$

Using classical homological algebra, we detect a kind of adjointness

$$
\mathfrak{C h h}\left(\boldsymbol{A}_{*} \otimes K_{*}, \boldsymbol{B}_{*}\right) \approx \mathfrak{C h} \mathfrak{h}\left(\boldsymbol{A}_{*}, \boldsymbol{B}_{*}^{K_{*}}\right), K_{*} \in \mathfrak{c h} ;
$$

hence, because $\boldsymbol{A}_{*} \otimes K_{*}, \boldsymbol{B}_{*} \in \mathfrak{C h}$ and since the embedding $\mathfrak{C h} \subset \mathfrak{C h} \mathfrak{h}$ is full, an isomorphism

$$
\mathfrak{C h}\left(\boldsymbol{A}_{*} \otimes K_{*}, \boldsymbol{B}_{*}\right) \approx \mathfrak{C h} \mathfrak{h}\left(\boldsymbol{A}_{*}, \boldsymbol{B}_{*}^{K_{*}}\right), K_{*} \in \mathfrak{c h}, \boldsymbol{A}_{*}, \boldsymbol{B}_{*} \in \mathfrak{C h}
$$

analogous to the special case when $K_{*}=C_{*}(K), K \in \mathfrak{S}$. Here we are not allowed to replace $\boldsymbol{B}_{*}^{K}$ by $\Phi\left(\boldsymbol{B}_{*}^{K}\right)$ and $\mathfrak{C h} \mathfrak{h}$ by $\mathfrak{C h}$.

Lemma 3.4. Let $j: K \subset L$ be a cofibration in $\mathfrak{S}$ (hence an inclusion). Then

$$
\boldsymbol{A}_{*}^{L} \stackrel{\boldsymbol{A}_{*}^{j}}{\longrightarrow} \boldsymbol{A}_{*}^{K}
$$

is a (regular, i.e. compatible with $\kappa, \varphi$ ) fibration in $\mathfrak{C h} \mathfrak{h}$.

Proof. We have

$$
\operatorname{Hom}\left(L_{p}, A_{p+n}(X, U)\right) \approx \operatorname{Hom}\left(K_{p}, A_{p+n} X, U\right) \oplus \operatorname{Hom}\left(C_{p}, A_{p+n}(X, U)\right),
$$

where $C_{p}$ is the free abelian group generated by the $p$-simplexes in $L \backslash K$. This is a natural decomposition, compatible with $l, i^{\prime}$. So we obtain

$$
\boldsymbol{A}_{*}^{L}(X, U) \approx \boldsymbol{A}_{*}^{K}(X, U) \oplus R_{*}(X, U)
$$

with appropriate $R_{*}(X, U)$. According to our definition of a fibration in $\mathfrak{C h} \mathfrak{h}$, this confirms that $j^{*}=\boldsymbol{A}_{*}^{j}$ is a regular fibration in $\mathfrak{C h} \mathfrak{h}$.

Lemma 3.5. Let $g \in \mathfrak{S}(K, L)$ be a weak equivalence; hence (according to the proof of 2.3 2)) $C_{*}(g) \in \mathfrak{c h}\left(C_{*}(K), C_{*}(L)\right)$ is a chain homotopy equivalence. Then, for any $\boldsymbol{A}_{*} \in \mathfrak{C h}$, the mappings $\boldsymbol{A}_{*}^{g} \in \mathfrak{C h} \mathfrak{h}\left(\boldsymbol{A}_{*}^{L}, \boldsymbol{A}_{*}^{K}\right)$ and $\Phi\left(\boldsymbol{A}_{*}^{g}\right) \in \mathfrak{C h}\left(\Phi\left(\boldsymbol{A}_{*}^{L}, \boldsymbol{A}_{*}\right)^{K}\right)$ are homotopy equivalences.

Proof. Immediate.

Corollary 3.6. Let $j: K \subset L$ be a (trivial) cofibration. Then $j^{*}=\Phi\left(\boldsymbol{A}_{*}^{j}\right) \in$ $\mathfrak{C h}\left(\Phi\left(\boldsymbol{A}_{*}^{l}\right), \Phi\left(\boldsymbol{A}_{*}^{K}\right)\right)$ is a (trivial) fibration.

Proposition 3.7. Let $j: K \subset L$ be a trivial cofibration in $\mathfrak{S}, \boldsymbol{C}_{*} \in \mathfrak{C h}$ and let

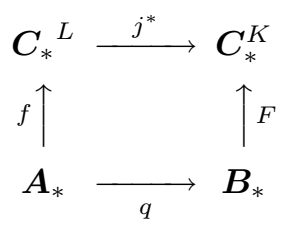

be commutative in $\mathfrak{C h h}$ with cofibration $q$ in $\mathfrak{C h}$.

Then there exists a diagonal $G: \boldsymbol{B}_{*} \longrightarrow \boldsymbol{C}_{*}^{L}$ rendering (6) commutative and a $\bar{G}: \boldsymbol{B}_{*} \otimes L \longrightarrow \boldsymbol{C}_{*}$ in $\mathfrak{C h}$, such that $\bar{G}\left(1_{\boldsymbol{B}_{*}} \otimes j\right)=\tilde{F}$ is the adjoint of $F$ in the sense of (5). 
Proof. Let $\hat{f}: \boldsymbol{A}_{*} \longrightarrow \Phi\left(\boldsymbol{C}_{*}^{L}\right), \tilde{F}: \boldsymbol{B}_{*} \longrightarrow \Phi\left(\boldsymbol{C}_{*}^{K}\right)$ be the mappings of Lemma 3.2. Then the diagram

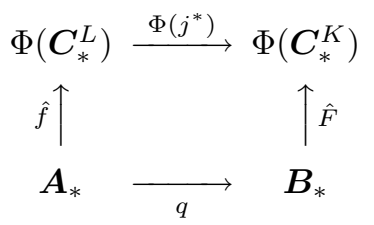

is commutative. Since, according to Lemma $3.4, j^{*}$ in (6) is a trivial fibration in $\mathfrak{C h h}$, Corollary 3.6 ensures that $\Phi\left(j^{*}\right)$ is a trivial fibration in $\mathfrak{C h}$. So there exists a diagonal $\overline{\hat{F}}: \boldsymbol{B}_{*} \longrightarrow \Phi\left(\boldsymbol{C}_{*}^{L}\right)$ rendering (7) commutative. Hence $\lambda \hat{F}=G$ and the adjoint of $G$ (in the sense of $(5))$ is a morphism in $\mathfrak{C h}$ satisfying $\tilde{G}(1 \otimes j)=\tilde{F}$.

In the same way as for tensor products, we may consider instead of $\boldsymbol{A}_{*}^{K}=\boldsymbol{A}_{*}^{C_{*}(K)}$, the chain complex functor $\boldsymbol{A}_{*}^{\tilde{C}_{*}(K)} \in \mathfrak{C h h}$. It is clear that for $K=I=\Delta^{1}$ this is isomorphic to $\boldsymbol{A}_{*}^{I}$ in the sense of [4], $\S 1$. We can treat homotopies $H: \boldsymbol{A}_{*} \longrightarrow \boldsymbol{B}_{*}^{\tilde{C}_{*}(I)}$ in $\mathfrak{C h}$ between mappings $f_{0}, f_{1}: \boldsymbol{A}_{*} \longrightarrow \boldsymbol{B}_{*}$ in the same way as we dealt with the left homotopies in $\S 2$. In particular we conclude that $f_{0} \simeq f_{1}$ if and only if we detect a right homotopy $\hat{H}: \boldsymbol{A}_{*} \longrightarrow \boldsymbol{B}_{*}^{C_{*}(I)}$ in $\mathfrak{C h h}$.

Suppose $K \in \mathfrak{S}$ is a finite simplicial set, i.e. it has only finitely many nondegenerate simplexes and $\boldsymbol{A}_{*} \in \mathfrak{C h}$. Then the direct product in the definition (1) of $\boldsymbol{A}_{*}^{\tilde{C}_{*}(K)}$ reduces to a direct sum. As a result $\boldsymbol{A}_{*}^{\tilde{C}_{*}(K)}$ becomes an object of $\mathfrak{C h}$.

We summarize:

Lemma 3.8. 1) Let $K \in \mathfrak{S}$ be a finite simplicial set and $\boldsymbol{A}_{*} \in \mathfrak{C h}$. Then $\boldsymbol{A}_{*}^{K}=$ $\boldsymbol{A}_{*}^{C_{*}(K)}$ is homotopy equivalent in $\mathfrak{C h h}$ to the chain functor $\boldsymbol{A}_{*}^{\tilde{C}_{*}(K)} \in \mathfrak{C h}$.

2) Two mappings $f_{0}, f_{1} \in \mathfrak{C h}\left(\boldsymbol{A}_{*}, \boldsymbol{B}_{*}\right)$ are homotopic whenever there exists an $\hat{H}: \boldsymbol{A}_{*} \longrightarrow \boldsymbol{B}_{*}^{C_{*}\left(\Delta^{1}\right)}$ in $\mathfrak{C h h}$ such that

$$
\boldsymbol{B}_{*}^{\partial_{k}} \hat{H}=f_{k}, \partial_{k}: \Delta^{0} \subset \Delta^{1}, k=0,1 .
$$

\section{Hom objects and adjointness}

We denote by $\Delta^{n}$ the simplicial set with simplex $\delta^{n}$ in dimension $n$ and $\partial_{i_{1}} \ldots$ $\partial_{i_{n-p}} \delta^{n}$ in lower dimensions. All other simplexes are degenerate. Let $\boldsymbol{A}_{*}, \boldsymbol{B}_{*} \in \mathfrak{C h}$. Then we define a simplicial set $\operatorname{Hom}\left(\boldsymbol{A}_{*}, \boldsymbol{B}_{*}\right)$ by setting

$$
\operatorname{Hom}\left(\boldsymbol{A}_{*}, \boldsymbol{B}_{*}\right)_{n}=\mathfrak{C h}\left(\boldsymbol{A}_{*} \otimes \Delta^{n}, \boldsymbol{B}_{*}\right) .
$$

Boundaries and degeneracies are induced by those of $\Delta^{n}$ (cf. [6], [7]).

Lemma 4.1. $\boldsymbol{H o m}\left(\boldsymbol{A}_{*}, \boldsymbol{B}_{*}\right)$ is a fibrant object in $\mathfrak{S}$ (i.e. a Kan set). Moreover

$$
\text { Hom: } \mathfrak{C h}^{o p} \times \mathfrak{C h} \longrightarrow \mathfrak{S}
$$

is a functor. 
Proof. The induced mappings are immediate. The fact that $\operatorname{Hom}\left(\boldsymbol{A}_{*}, \boldsymbol{B}_{*}\right)$ is fibrant will be a consequence of 4.4 (2). However it can be deduced directly by considering the fibration $p: \boldsymbol{B}_{*} \longrightarrow\{0\}$ (cf. [3], $1.1(3)$ ) in the commutative diagram

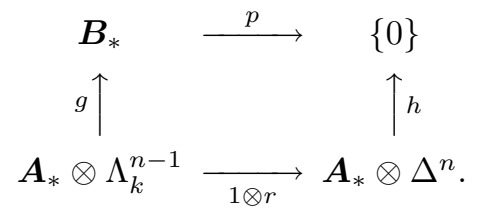

Here $\Lambda_{k}^{n-1}$ is a horn in $\Delta^{n}$ (i.e., all $n-1$ boundary simplexes with the exception of $\left.\partial_{k} \delta^{n}\right) ; r: \Lambda_{k}^{n-1} \longrightarrow \Delta^{n}$ is the inclusion (being a trivial cofibration, implying that so is $1 \otimes r)$ and $g$ a horn in $\operatorname{Hom}\left(\boldsymbol{A}_{*}, \boldsymbol{B}_{*}\right)$, with filling $h$ of the image of $g$ (i.e. of $p g)$. The diagonal $G: \boldsymbol{A}_{*} \otimes \Delta^{n} \longrightarrow \boldsymbol{B}_{*}$ exhibits a filling of $g$, confirming that

$$
\operatorname{Hom}\left(\boldsymbol{A}_{*}, \boldsymbol{B}_{*}\right) \longrightarrow \operatorname{Hom}\left(\boldsymbol{A}_{*},\{0\}\right)
$$

is a fibration in $\mathfrak{S}$.

We obtain the following adjointness:

Theorem 4.2. There exists a natural (with respect to all variables involved) isomorphism

$$
\mathfrak{S}\left(K, \boldsymbol{H o m}\left(\boldsymbol{A}_{*}, \boldsymbol{B}_{*}\right)\right) \approx \mathfrak{C h}\left(\boldsymbol{A}_{*} \otimes K, \boldsymbol{B}_{*}\right) \text { for } K \in \mathfrak{S} .
$$

Proof. Suppose $K \in \mathfrak{S}, \boldsymbol{A}_{*}, \boldsymbol{B}_{*} \in \mathfrak{C h}$. Then $\alpha \in \mathfrak{S}\left(K, \operatorname{Hom}\left(\boldsymbol{A}_{*}, \boldsymbol{B}_{*}\right)\right)$ is an assignment

$$
\alpha\left(\sigma^{n}\right)=\left(\boldsymbol{A}_{*} \otimes \Delta^{n} \longrightarrow \boldsymbol{B}_{*}\right), \quad \sigma^{n} \in \mathfrak{S},
$$

which commutes with boundaries and degeneracies. So $\alpha\left(\sigma^{n}\right)\left(a^{p} \otimes \delta^{q}\right) \in B_{p+q}$. On the other hand, $\beta \in \mathfrak{C h}\left(\boldsymbol{A}_{*} \otimes K, \boldsymbol{B}_{*}\right)$ is an assignment $\beta\left(a^{p} \otimes \sigma^{q}\right) \in B_{p+q}$.

To each $\alpha$ we associate $\tilde{\alpha} \in \mathfrak{C h}\left(\boldsymbol{A}_{*} \otimes K, B_{*}\right)$ by

$$
\tilde{\alpha}\left(a^{p} \otimes \sigma^{q}\right)=\alpha\left(\sigma^{q}\right)\left(a^{p} \otimes \delta^{q}\right) .
$$

Suppose $\beta \in \mathfrak{C h}\left(\boldsymbol{A}_{*} \otimes K, \boldsymbol{B}_{*}\right)$. Then we set

$$
\tilde{\beta}\left(\sigma^{q}\right)\left(a^{p} \otimes \delta^{p}\right)=\beta\left(a^{p} \otimes \sigma^{q}\right) .
$$

We deduce that

$$
\tilde{\tilde{\alpha}}\left(\sigma^{q}\right)\left(a^{p} \otimes \delta^{q}\right)=\tilde{\alpha}\left(a^{p} \otimes \sigma^{q}\right)=\alpha\left(a^{p} \otimes \delta^{q}\right)
$$

and

$$
\tilde{\tilde{\beta}}\left(a^{p} \otimes \sigma^{q}\right)=\tilde{\beta}\left(\sigma^{q}\right)\left(a^{p} \otimes \delta^{q}\right)=\beta\left(a^{p} \otimes \sigma^{q}\right) .
$$

This yields an isomorphism (2) that is natural in all variables.

For a better understanding of $\operatorname{Hom}\left(\boldsymbol{A}_{*}, \boldsymbol{B}_{*}\right)$ we need:

Lemma 4.3. 1) Let $f_{o}, \simeq f_{1}: \boldsymbol{A}_{*} \longrightarrow \boldsymbol{C}_{*}$ be homotopic in $\mathfrak{C h}$. Then the induced mappings $f_{0}^{*}, f_{1}^{*}: \boldsymbol{H o m}\left(\boldsymbol{C}_{*}, \boldsymbol{B}_{*}\right) \longrightarrow \boldsymbol{H o m}\left(\boldsymbol{A}_{*}, \boldsymbol{B}_{*}\right)$ are homotopic in $\mathfrak{S}$. 
2) Let $_{g_{0}}, g_{1}: \boldsymbol{B}_{*} \longrightarrow \boldsymbol{C}_{*}$ be homotopic in $\mathfrak{C h}$. Then $g_{0 *}, g_{1 *}: \boldsymbol{H o m}\left(\boldsymbol{A}_{*}, \boldsymbol{B}_{*}\right) \longrightarrow$ $\boldsymbol{H o m}\left(\boldsymbol{A}_{*}, \boldsymbol{C}_{*}\right)$ are homotopic in $\mathfrak{S}$.

Proof. 1) We have a homotopy $H: f_{0} \simeq f_{1}, H: \boldsymbol{A}_{*} \otimes \Delta^{1} \longrightarrow \boldsymbol{C}_{*}$ (cf. Lemma 1.2 2)); hence a map

$$
H^{*}: \operatorname{Hom}\left(\boldsymbol{C}_{*}, \boldsymbol{B}_{*}\right) \longrightarrow \operatorname{Hom}\left(\boldsymbol{A}_{*} \otimes \Delta^{1}, \boldsymbol{B}_{*}\right)
$$

satisfying $\delta_{i}^{*} H^{*}=f_{i}^{*}$ for $i=0,1$ where $\delta_{i}: \boldsymbol{A}_{*} \longrightarrow \boldsymbol{A}_{*} \otimes \Delta^{1}$ and

$$
\delta_{i}^{*}: \operatorname{Hom}\left(\boldsymbol{A}_{*} \otimes \Delta^{1}, \boldsymbol{B}_{*}\right) \longrightarrow \operatorname{Hom}\left(\boldsymbol{A}_{*}, \boldsymbol{B}_{*}\right) .
$$

By the definition of a function complex in $\mathfrak{S}$, we have (cf. [6], Ch. 1)

$$
\left(K^{L}\right)_{n}=\mathfrak{S}\left(L \times \Delta^{n}, K\right) .
$$

So we conclude:

$$
\begin{gathered}
\operatorname{Hom}\left(\boldsymbol{A}_{*} \otimes \Delta^{1}, \boldsymbol{B}_{*}\right)_{n}=\mathfrak{C h}\left(\left(\boldsymbol{A}_{*} \otimes \Delta^{1}\right) \otimes \Delta^{n}, \boldsymbol{B}_{*}\right) \cong \mathfrak{C h}\left(\boldsymbol{A}_{*} \otimes\left(\Delta^{1} \times \Delta^{n}\right), \boldsymbol{B}_{*}\right) \\
\approx \mathfrak{S}\left(\Delta^{1} \times \Delta^{n}, \boldsymbol{H o m}\left(\boldsymbol{A}_{*} \cdot \boldsymbol{B}_{*}\right)\right) \approx\left(\operatorname{Hom}\left(\boldsymbol{A}_{*}, \boldsymbol{B}_{*}\right)^{\Delta^{1}}\right)_{n} .
\end{gathered}
$$

Therefore there exists a natural homotopy equivalence by adjunction

$$
\theta^{k}: \operatorname{Hom}\left(\boldsymbol{A}_{*} \otimes \Delta^{k}, \boldsymbol{B}_{*}\right) \cong \operatorname{Hom}\left(\boldsymbol{A}_{*}, \boldsymbol{B}_{*}\right)^{\Delta^{k}}, k=0,1 .
$$

To complete the proof, we need the following commutative diagram

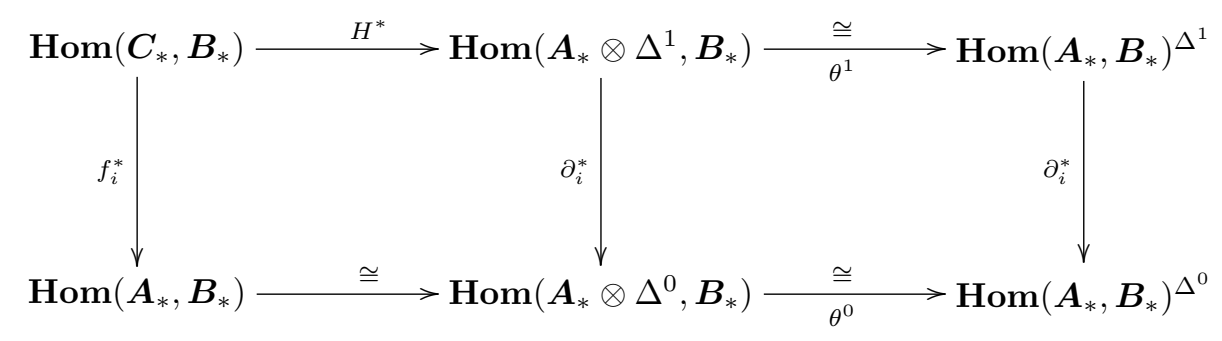

where $\partial_{i}^{*}$ are both induced by the mappings $\partial_{i}: \Delta^{0} \subset \Delta^{1}$. The commutativity follows from the naturality of $\theta$.

This completes the proof that $f_{0}^{*} \simeq f_{1}^{*}$.

2) There exists a homotopy $G: \boldsymbol{B}_{*} \longrightarrow \boldsymbol{C}_{*}{ }^{\Delta^{1}}$ between $g_{0}$ and $g_{1}$; hence we have $p_{i} G=g_{i}, i=0,1$, where $p_{i}: \boldsymbol{C}_{*} \Delta^{1} \longrightarrow \boldsymbol{C}_{*}$ are the projections. Now we argue as in the first case:

$$
\operatorname{Hom}\left(\boldsymbol{A}_{*}, \boldsymbol{C}_{*} \Delta^{1}\right) \approx \operatorname{Hom}\left(\boldsymbol{A}_{*} \otimes \Delta^{1}, \boldsymbol{C}_{*}\right) \cong \operatorname{Hom}\left(\boldsymbol{A}_{*}, \boldsymbol{C}_{*}\right)^{\Delta^{1}}
$$

and obtain a homotopy commutative diagram

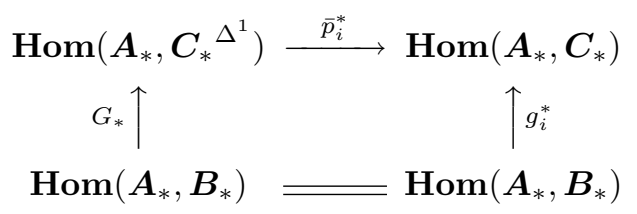

which furnishes a homotopy $g_{o *} \simeq g_{1 *}$. 
A horn $\tilde{\Lambda}_{k}^{n-1}$ in some $\operatorname{Hom}\left(\boldsymbol{A}_{*}, \boldsymbol{B}_{*}\right)$ is a collection of $(n-1)$ simplexes $\sigma_{0}^{n-1}, \ldots$, $\hat{\sigma}_{k}^{n-1}, \ldots, \sigma_{n}^{n-1}$ which fit together at the boundaries like the boundary simplexes of an $n$-simplex, with the exception of $\sigma_{k}^{n-1}$. Hence, we can identify $\tilde{\Lambda}_{k}^{n-1}$ with a mapping

$$
g: \boldsymbol{A}_{*} \otimes \Lambda_{k}^{n-1} \longrightarrow \boldsymbol{B}_{*}
$$

and a filling $\tilde{\Delta}^{n}$ of $\tilde{\Lambda}_{k}^{n-1}$ with a $G: \boldsymbol{A}_{*} \otimes \Delta^{n} \longrightarrow \boldsymbol{B}_{*}$, agreeing with $g$ on $\Lambda_{k}^{n-1}$. This observation will be crucial for the proof of 1) and 2) of the following lemma.

Lemma 4.4. 1) Let $q: \boldsymbol{A}_{*} \subset \boldsymbol{B}_{*}$ be a cofibration in $\mathfrak{C h}$. Then $q^{*}: \boldsymbol{H o m}\left(\boldsymbol{B}_{*}, \boldsymbol{C}_{*}\right)$ $\longrightarrow \boldsymbol{H o m}\left(\boldsymbol{A}_{*}, \boldsymbol{C}_{*}\right)$ is a fibration in $\mathfrak{S}$.

2) Let $p: \boldsymbol{A}_{*} \longrightarrow \boldsymbol{B}_{*}$ be a fibration in $\mathfrak{C h}$. Then

$$
p_{*}: \boldsymbol{H o m}\left(\boldsymbol{C}_{*}, \boldsymbol{A}_{*}\right) \longrightarrow \boldsymbol{H o m}\left(\boldsymbol{C}_{*}, \boldsymbol{B}_{*}\right)
$$

is a fibration in $\mathfrak{S}$.

3) Let $s: \boldsymbol{A}_{*} \longrightarrow \boldsymbol{B}_{*}$ be a weak equivalence in $\mathfrak{C h}$. Then

$$
p_{*}: \operatorname{Hom}\left(\boldsymbol{B}_{*}, \boldsymbol{C}_{*}\right) \longrightarrow \boldsymbol{H o m}\left(\boldsymbol{A}_{*}, \boldsymbol{C}_{*}\right)
$$

is a homotopy equivalence in $\mathfrak{S}$.

4) Let $t: \boldsymbol{A}_{*} \longrightarrow \boldsymbol{B}_{*}$ be a weak equivalence in $\mathfrak{C h}$. Then

$$
t_{*}: \operatorname{Hom}\left(\boldsymbol{C}_{*}, \boldsymbol{A}_{*}\right) \longrightarrow \boldsymbol{H o m}\left(\boldsymbol{C}_{*}, \boldsymbol{B}_{*}\right)
$$

is a homotopy equivalence in $\mathfrak{S}$.

Proof. 1) The commutative diagram

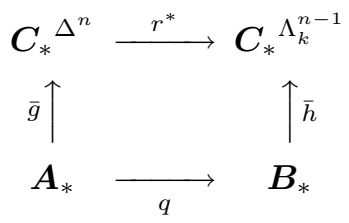

expresses the fact that a horn $h(q \otimes 1): \boldsymbol{A}_{*} \otimes \Lambda_{k}^{n-1} \longrightarrow \boldsymbol{C}_{*}$ has a filling $g: \boldsymbol{A}_{*} \otimes$ $\Delta^{n} \longrightarrow \boldsymbol{C}_{*}\left(h: \boldsymbol{B}_{*} \otimes \Lambda_{k}^{n-1} \longrightarrow \boldsymbol{C}_{*}\right.$ being itself a horn). Since $r: \Lambda_{k}^{n-1} \subset \Delta^{n}$ is a trivial cofibration, we can apply 3.7 to the effect that there exists a diagonal $G: \boldsymbol{B}_{*} \otimes$ $\Delta^{n} \longrightarrow C_{*}$ displaying a filling of the original horn $h$, mapping in the expected way.

2) Now we consider the commutative square

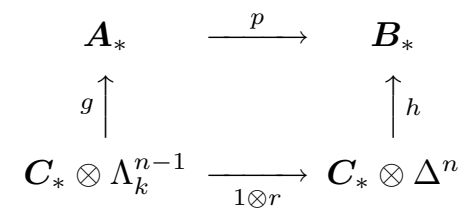

where $g$ is a given horn in $\operatorname{Hom}\left(\boldsymbol{C}_{*}, \boldsymbol{A}_{*}\right)$ and $h$ a filling of the image of this horn under $p_{*}: \operatorname{Hom}\left(\boldsymbol{C}_{*}, \boldsymbol{A}_{*}\right) \longrightarrow \operatorname{Hom}\left(\boldsymbol{C}_{*}, \boldsymbol{B}_{*}\right)$. Since $1 \otimes r$ is a trivial cofibration (cf. 
Lemma 2.2) we detect a lifting

$$
G: \boldsymbol{C}_{*} \otimes \Delta^{n} \longrightarrow \boldsymbol{A}_{*},
$$

which is a filling of $g$ which is mapped under $p$ into $h$.

$3), 4)$ These follow immediately from Lemma 4.3.

Proposition 4.5. There exists a composition

$$
\operatorname{Hom}\left(B_{*}, C_{*}\right) \times \operatorname{Hom}\left(A_{*}, B_{*}\right) \longrightarrow \operatorname{Hom}\left(A_{*}, C_{*}\right)
$$

which is associative and has identities as in [6], Ch. 2 §2, i.e., $\mathfrak{C h}$ is an enrichment over the category $\mathfrak{S}$.

Proof. The proof follows entirely the same pattern as in Ch. 2 of [6] adapted to our present situation.

Suppose $g: \boldsymbol{B}_{*} \otimes \triangle^{n} \longrightarrow \boldsymbol{C}_{*}$ and $f: \boldsymbol{A}_{*} \otimes \triangle^{n} \longrightarrow \boldsymbol{B}_{*}$ are given $n$-simplexes in these simplicial sets. Then we define $h: \boldsymbol{A}_{*} \otimes \triangle^{n} \longrightarrow \boldsymbol{C}_{*}$ by

$$
\boldsymbol{A}_{*} \otimes \triangle^{n} \stackrel{1 \otimes d}{\longrightarrow} \boldsymbol{A}_{*} \otimes\left(\triangle^{n} \times \triangle^{n}\right) \cong\left(\boldsymbol{A}_{*} \otimes \triangle^{n}\right) \otimes \triangle^{n} \stackrel{f \otimes \triangle^{n}}{\longrightarrow} \boldsymbol{B}_{*} \otimes \triangle^{n} \stackrel{g}{\longrightarrow} \boldsymbol{C}_{*}
$$

where $\cong$ denotes now an associative EZ-homotopy equivalence (cf. $\S 2(3)$ and (4)) and $d$ is the diagonal map.

Concerning the associativity of this composition law, we consider

$$
f \in \boldsymbol{H o m}\left(\boldsymbol{A}_{*}, \boldsymbol{B}_{*}\right)_{n}, g \in \boldsymbol{H o m}\left(\boldsymbol{B}_{*}, \boldsymbol{C}_{*}\right)_{n} \text { and } h \in \operatorname{Hom}\left(\boldsymbol{C}_{*}, \boldsymbol{D}_{*}\right)_{n}
$$

hence $f: \boldsymbol{A}_{*} \otimes \triangle^{n} \longrightarrow \boldsymbol{B}_{*}, g: \boldsymbol{B}_{*} \otimes \triangle^{n} \longrightarrow \boldsymbol{C}_{*}, h: \boldsymbol{C}_{*} \otimes \triangle^{n} \longrightarrow \boldsymbol{D}_{*}$. Comparing $(h g) f$ with $h(g f)$ amounts to checking the commutativity of a large but immediately established diagram. It turns out that this diagram is commutative, hence that $(h g) f=h(g f)$ whenever the diagram $\S 2(4)$ is commutative, which can be accomplished by choosing the appropriate EZ-map, as we pointed out in $\S 2$. This settles the associativity of the composition law.

The existence of the identities follows entirely as in [6], Ch. $2 \S 2$.

The next assertion is one of the basic properties of a tensored category. In our case it follows more or less immediately from Theorem 4.2.

Theorem 4.6. There exists a natural homotopy equivalence of simplicial sets

$$
\boldsymbol{H o m}\left(\boldsymbol{A}_{*} \otimes K, \boldsymbol{B}_{*}\right) \cong \boldsymbol{H o m}\left(K, \boldsymbol{H o m}\left(\boldsymbol{A}_{*}, \boldsymbol{B}_{*}\right)\right)
$$

which is induced by the EZ-map $§ 2$ (3).

Proof. According to the various definition of function complexes and according to 
(2) we conclude:

$$
\begin{gathered}
\operatorname{Hom}\left(\boldsymbol{A}_{*}, \boldsymbol{B}_{*}\right)_{n}=\mathfrak{C h}\left(\boldsymbol{A}_{*} \otimes \triangle^{n}, \boldsymbol{B}_{*}\right) \\
\operatorname{Hom}\left(K, \operatorname{Hom}\left(\boldsymbol{A}_{*}, \boldsymbol{B}_{*}\right)\right)_{n}=\mathfrak{S}\left(K \times \triangle^{n}, \operatorname{Hom}\left(\boldsymbol{A}_{*}, \boldsymbol{B}_{*}\right)\right) \\
\operatorname{Hom}\left(\boldsymbol{A}_{*} \otimes K, \boldsymbol{B}_{*}\right)_{n}=\mathfrak{C h}\left(\left(\boldsymbol{A}_{*} \otimes K\right) \otimes \triangle^{n}, \boldsymbol{B}_{*}\right) \stackrel{\cong}{\longrightarrow} \\
\mathfrak{C h}\left(\boldsymbol{A}_{*} \otimes\left(K \times \triangle^{n}\right), \boldsymbol{B}_{*}\right) \approx \mathfrak{S}\left(K, \operatorname{Hom}\left(\boldsymbol{A}_{*}, \boldsymbol{B}_{*}\right)\right)_{n},
\end{gathered}
$$

where $\cong$ refers to a homotopy equivalence, induced by $\S 2(3)$. Since all these mappings commute with boundary and degeneracy operators, this yields a homotopy equivalence (6).

Remark 4.7. In the presence of $\S 2(3)$ the homotopy equivalence (6) follows immediately from (2) (which is the 0-level of (6)). The homotopy equivalence in (6) is of the same kind as the equivalence in $\S 2(3)$ which directly induces (6).

\section{The axiom SM7 for $\otimes$-products}

There are several formulations of D. Quillen's axiom SM7 (cf. [6], [7]). In this section we deal with the $\otimes$-product version:

SM7: Let $q: \boldsymbol{A}_{*} \subset \boldsymbol{B}_{*}$ in $\mathfrak{C h}$ and $j: K \subset L$ in $\mathfrak{S}$ be cofibrations. Then there exists a pushout

$$
\boldsymbol{P}_{*}=\boldsymbol{A}_{*} \otimes L \cup_{\boldsymbol{A}_{*} \otimes K} \boldsymbol{B}_{*} \otimes K
$$

in $\mathfrak{C h}$ and a mapping

$$
w=\left(\boldsymbol{B}_{*} \otimes j, q \otimes L\right): \boldsymbol{P}_{*} \longrightarrow \boldsymbol{B}_{*} \otimes L
$$

which is a cofibration. Moreover $w$ is a trivial cofibration whenever $q$ or $j$ is a trivial cofibration.

To be more precise: $\boldsymbol{P}_{*}$ is the pushout in the diagram

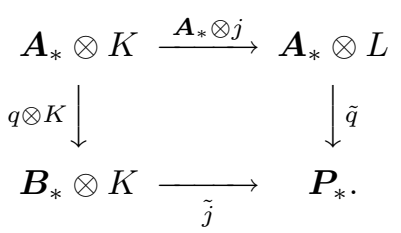

According to Lemmas 2.2 and 2.4, $\boldsymbol{A}_{*} \otimes j$ and $q \otimes K$ are cofibrations. Theorem 3.2 in [3] ensures that $\boldsymbol{P}_{*}$ is a chain functor (hence this pushout exists in $\mathfrak{C h}$ ).

Lemma 5.1. $w$ is a cofibration.

Proof. Firstly, $w$ is an inclusion, hence commuting with $i^{\prime}, l, \varphi, \kappa$. Since $q$ and $j$ are cofibrations, we have levelwise retractions $\hat{q}: B_{n} \longrightarrow A_{n}, s:\left(B_{*} \otimes L\right)_{n} \longrightarrow$ $\left(B_{*} \otimes K\right)_{n}, t=\hat{q} \otimes L:\left(B_{*} \otimes L\right)_{n} \longrightarrow\left(A_{*} \otimes L\right)_{n}, n \in \mathbb{Z}$, for $q, \boldsymbol{B}_{*} \oplus j$ and $q \otimes L$ respectively. 
Let $\tau: \boldsymbol{B}_{*} \otimes K \bigoplus \boldsymbol{A}_{*} \otimes L \longrightarrow \boldsymbol{P}_{*}$ be the natural surjection and $\rho: \boldsymbol{B}_{*} \otimes K \longrightarrow$ $\boldsymbol{B}_{*} \otimes K \bigoplus \boldsymbol{A}_{*} \otimes L \longleftarrow \boldsymbol{A}_{*} \otimes L: \rho^{\prime}$ the coproduct injections. We have

$$
\begin{gathered}
\tilde{q} t w \tilde{q}=\tilde{q} t(q \otimes L)=\tilde{q} \\
\tilde{j} s w \tilde{j}=\tilde{j} s\left(1_{\boldsymbol{B}_{*}} \otimes j\right)=\tilde{j},
\end{gathered}
$$

where $\tilde{q}, \tilde{j}$ are from (1).

We claim that $\tau(t, s)$ is a levelwise, natural retraction for $w$; hence

$$
\tau(t, s) w=1
$$

(where $(t, s)=\rho^{\prime} t+\rho s$ ), implying that $w$ is a cofibration. Due to the universality of a pushout, it is sufficient to verify that

$$
\tau(t, s) w \tilde{q}=\tilde{q}, \tau(t, s) w \tilde{j}=\tilde{j} .
$$

Since $\tau \rho^{\prime}=\tilde{q}, \tau \rho=\tilde{j}$, this follows from (2), (3), the commutativity of (1) and the fact that

$$
\tilde{j} s w \tilde{q}\left|\left(\boldsymbol{B}_{*} \otimes K \backslash \boldsymbol{A}_{*} \otimes K\right)=0, \tilde{q} t w \tilde{j}\right|\left(\boldsymbol{A}_{*} \otimes L \backslash \boldsymbol{A}_{*} \otimes K\right)=0 .
$$

This completes the proof of Lemma 5.1.

Lemma 5.2. 1) Let $q: \boldsymbol{A}_{*} \subset \boldsymbol{B}_{*}$ be a trivial cofibration in $\mathfrak{C h}$. Then $w$ is a trivial cofibration.

2) Let $j: K \subset L$ be a trivial cofibration in $\mathfrak{S}$. Then $w$ is a trivial cofibration in $\mathfrak{C h}$.

Proof. First, observe that in (1) $w \tilde{q}=q \otimes 1_{L}, w \tilde{j}=1_{\boldsymbol{B}_{*}} \otimes j$.

1) If $q$ is a trivial cofibration, then $q \otimes 1_{K}, q \otimes 1_{L}$ and $\tilde{q}$ are trivial cofibrations (2.5 (2), 2.6 (1)). Hence by the two-out-of-three axiom (Quillen's CM2), cf. [6], [7], [11]) $w$ is a trivial cofibration.

2) If $j$ is a trivial cofibration, then $1_{\boldsymbol{A}_{*}} \otimes j, 1_{\boldsymbol{B}_{*}} \otimes j$ and $\tilde{j}$ are trivial cofibrations (2.5 (1), 2.6). Again, CM2 confirms that $w$ is a trivial cofibration.

We summarize:

Theorem 5.3. The model structure of $\mathfrak{C h}$ (cf. [4]) together with the $\otimes$-product depicted in $§ 1$ satisfies axiom SM\%.

\section{SM7 for Hom}

In a model structure, different formulations of SM7 turn out to be equivalent (cf. [6], [7]). However since $\mathfrak{C h}$ does not have all (co-)limits which are needed for proving such an equivalence, we present an independent proof for SM7 in the Homformulation: 
Suppose $q: \boldsymbol{A}_{*} \longrightarrow \boldsymbol{B}_{*}$ is a cofibration and $p: \boldsymbol{X}_{*} \longrightarrow \boldsymbol{Y}_{*}$ a regular fibration in $\mathfrak{C h}$. We consider the pullback diagram in $\mathfrak{S}$ :

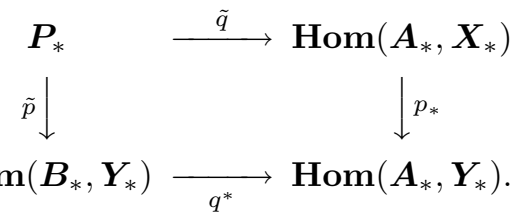

$\boldsymbol{P}_{*} \subset \boldsymbol{H o m}\left(\boldsymbol{A}_{*}, \boldsymbol{X}_{*}\right) \times \operatorname{Hom}\left(\boldsymbol{B}_{*}, \boldsymbol{Y}_{*}\right)$ and the mapping

$$
w: \operatorname{Hom}\left(\boldsymbol{B}_{*}, \boldsymbol{X}_{*}\right) \longrightarrow \boldsymbol{P}_{*}
$$

are defined and determined by the properties

$$
\begin{aligned}
& \operatorname{Hom}\left(q, \boldsymbol{X}_{*}\right)=\tilde{q} w \\
& \operatorname{Hom}\left(\boldsymbol{B}_{*}, p\right)=\tilde{p} w .
\end{aligned}
$$

In this case, the existence of $\boldsymbol{P}_{*} \in \mathfrak{S}$ is not a problem, but we have to verify:

Proposition 6.1. $w$ is a fibration in $\mathfrak{S}$.

Proof. Suppose we have a horn in $\operatorname{Hom}\left(\boldsymbol{B}_{*}, \boldsymbol{X}_{*}\right)$, hence an $f: \boldsymbol{B}_{*} \otimes \Lambda_{k}^{n-1} \longrightarrow \boldsymbol{X}_{*}$ and two fillings $g: \boldsymbol{A}_{*} \otimes \Delta^{n} \longrightarrow \boldsymbol{X}_{*}, h: \boldsymbol{B}_{*} \otimes \Delta^{n} \longrightarrow \boldsymbol{Y}_{*}$ (of $f q \otimes 1_{\Lambda_{k}^{n-1}}$ and $p f$ resp.) such that $q^{*}(h)=p_{*}(g)$ in $\operatorname{Hom}\left(\boldsymbol{A}_{*}, \boldsymbol{Y}_{*}\right)$. A pair with this property is a filling of $w(f)$ in $\boldsymbol{P}_{*}$. This amounts to the existence of the commutative diagrams

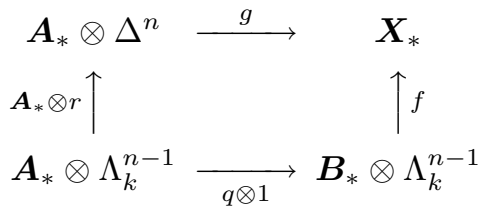

in $\mathfrak{C h h}$ (cf. $\S 3)$ and

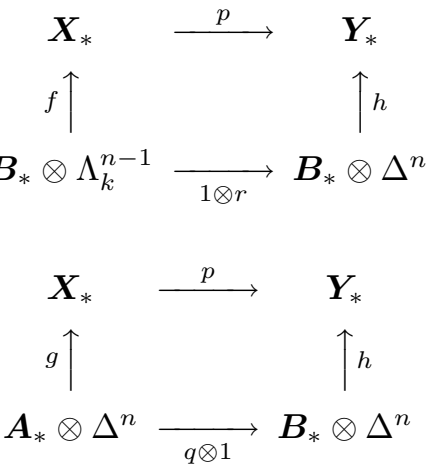

in $\mathfrak{C h}$. 
Homology, Homotopy and Applications, vol. 9(1), 2007

According to Lemma 4.41$), 2) \boldsymbol{H} \boldsymbol{o m}\left(q, \boldsymbol{X}_{*}\right), \boldsymbol{H} \boldsymbol{o m}\left(\boldsymbol{B}_{*}, p\right)$ are fibrations. Therefore there exist two fillings of $f$,

$$
F_{1}, F_{2}: \boldsymbol{B}_{*} \otimes \Delta^{n} \longrightarrow \boldsymbol{X}_{*}
$$

with

$$
\begin{gathered}
F_{1}(q \otimes 1)=g, \\
p F_{2}=h \\
F_{1}\left(\boldsymbol{B}_{*} \otimes r\right)=F_{2}\left(\boldsymbol{B}_{*} \otimes r\right)=f .
\end{gathered}
$$

However we do not know that $F_{1}=F_{2}$.

Because

$$
h(q \otimes 1)=p g,
$$

we obtain

$$
p F_{1}(q \otimes 1)=p F_{2}(q \otimes 1)
$$

hence

$$
p\left(F_{1}-F_{2}\right)(q \otimes 1)=0 .
$$

Since according to [3], Theorem 3.1 there exists a cokernel $\boldsymbol{B}_{*} / \boldsymbol{A}_{*}=\boldsymbol{C}_{*}$ of $q$, we detect (because the $\otimes$-product is a right exact functor) a $D: \boldsymbol{C}_{*} \otimes \Delta^{n} \longrightarrow \boldsymbol{Y}_{*}$ such that $p\left(F_{1}-F_{2}\right)=D \rho, \rho: \boldsymbol{B}_{*} \otimes \Delta^{n} \longrightarrow \boldsymbol{B}_{*} / \boldsymbol{A}_{*} \otimes \Delta^{n}=\boldsymbol{C}_{*} \otimes \Delta^{n}$. There exists a commutative diagram

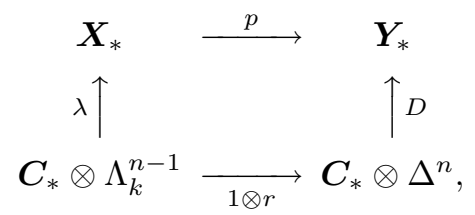

where $\lambda$ is induced by $F_{1}-F_{2}$, since

$$
\left(F_{1}-F_{2}\right)\left(\boldsymbol{B}_{*} \otimes r\right)\left(q \otimes 1_{\Lambda_{k}^{n-1}}\right)=(f-f)\left(q \otimes 1_{\Lambda_{k}^{n-1}}\right)=0 .
$$

We find in (7) a diagonal $\bar{D}: \boldsymbol{C}_{*} \otimes \Delta^{n} \longrightarrow \boldsymbol{X}_{*}$, with $p \bar{D}=D$ and set

$$
F=F_{1}-\bar{D} \rho \text {. }
$$

We calculate:

$$
\begin{gathered}
p F=p F_{1}-p \bar{D} \rho=p F_{1}-D \rho=p F_{2}=h \\
F\left(q \otimes 1_{\Delta^{n}}\right)=F_{1}\left(q \otimes 1_{\Delta^{n}}\right)-\bar{D} \rho\left(q \otimes 1_{\Delta^{n}}\right)=F_{1}\left(q \otimes 1_{\Delta^{n}}\right)=g \\
F \mid \boldsymbol{B}_{*} \otimes \Lambda_{k}^{n-1} \\
=F_{1}\left|\boldsymbol{B}_{*} \otimes \Lambda_{k}^{n-1}-\bar{D} \rho\right| \boldsymbol{B}_{*} \otimes \Lambda_{k}^{n-1} \\
=f-\left(F_{1}-F_{2}\right) \mid \boldsymbol{B}_{*} \otimes \Lambda_{k}^{n-1} \\
=f
\end{gathered}
$$

Hence $F: \boldsymbol{B}_{*} \otimes \Delta^{n} \longrightarrow \boldsymbol{X}_{*}$ is a filling of the given horn $f$ which is mapped into a given filling under $w$ in $\boldsymbol{P}_{*}$. 
This confirms that $w$ is a fibration (hence a Kan fibration) in $\mathfrak{S}$.

We summarize:

Theorem 6.2 (SM7). The mapping $w$ is a fibration in $\mathfrak{S}$, hence a Kan fibration, which is trivial, if either $q$ is a trivial cofibration or $p$ is a trivial fibration.

Proof. Let $q$ be a trivial cofibration. Then $\boldsymbol{H o m}\left(q, \boldsymbol{X}_{*}\right), \boldsymbol{H} \boldsymbol{o m}\left(q, \boldsymbol{Y}_{*}\right)$ and $\tilde{q}$ are trivial fibrations $(4.41), 3)$ and 2.62$)$ ). So we can apply the Quillen axiom CM2) for $\mathfrak{C h}$ to $(2)$, confirming that $w$ is a weak equivalence.

Suppose $p$ is a trivial fibration. Then $\boldsymbol{H o m}\left(\boldsymbol{X}_{*}, p\right), \boldsymbol{H o m}\left(\boldsymbol{Y}_{*}, p\right)$ and $\tilde{p}$ are trivial fibrations in $\mathfrak{S}(4.42), 4)$ and 2.62$)$ ). Again $w$ is recognized as a trivial fibration by applying CM2) and (3).

\section{7. ss-model structure}

It turns out that in the definition of a simplicial model structure, one can replace the category $\mathfrak{S}$ by suitable monoidal categories of spectra (such as the category of symmetric spectra or the category of S-modules), establishing highly structured model structures on the categories in question. For details concerning this well developed theory we refer to [8], Ch. 4.

Although we are not going to pursue this further for the category $\mathfrak{C h}$, we will in this section try to investigate what happens if instead of $\mathfrak{S}$, one deals with the stable category $\mathfrak{S p}$ of simplicial spectra.

According to [9], [10] a simplicial spectrum $X=\left\{X_{(q)}\right\}$ is a family of sets with basepoint $*, q \in \mathbb{Z}$, together with boundary and degeneracy operators $\partial_{i}: X_{(q)} \longrightarrow$ $X_{(q-1)}, s_{i}: X_{(q)} \longrightarrow X_{(q+1)}, i \geqslant 0$, satisfying the well-known identities for simplicial sets and, in addition, the requirement that only finitely many $\partial_{i} \sigma, \sigma \in X_{(q)}$ be different from the basepoint. The elements $\sigma^{q} \in X_{(q)}$ are called simplexes of degree $q$. The category of simplicial spectra is denoted by $\mathfrak{S p}$. The advantage of this category is its resemblance to the category of simplicial sets. In particular, the subcategories of (abelian) group spectra play the same role as the corresponding subcategories of simplicial sets.

In [10], to each $X \in \mathfrak{S p}$ is associated a family of simplicial sets $X_{p}, p \in \mathbb{Z}$, in the following way: An $n$-simplex $\eta^{n} \in\left(X_{p}\right)_{n}$ is a $\sigma^{n-p} \in X_{(n-p)}$, satisfying $\partial_{0} \cdots \partial_{n} \sigma^{n-p}$ $=*, \partial_{i} \sigma^{n-p}=*, i>n$. It turns out that the family of simplicial sets $\left\{X_{p}\right\}$ forms a prespectrum, i.e. there are mappings in $\mathfrak{S}$

$$
\Sigma X_{p} \longrightarrow X_{p+1}
$$

(cf. $[10], \S 2)$.

It turns out that one can replace in the definition of a simplicial model category the category $\mathfrak{S}$ by the category $\mathfrak{S p}$ :

Definition 7.1. If for a given model category $\mathfrak{L}$, the category $\mathfrak{S}$ in the definition of a simplicial model structure is replaced by the category $\mathfrak{S p}$, one obtains what we call a simplicial stable model structure (= ss-model structure). 
In accordance with our terminology in $\S 11$, we are able to talk about an approximate ss-model structure on the category $\mathfrak{C h}$.

Without going into the details again. we are able to replace $\mathfrak{S}$ in the construction of the modified SM6, SM7 simplicial sets by simplicial spectra $\mathfrak{S p}$. We recall that $\boldsymbol{A}_{*} \otimes K$ is defined by $\boldsymbol{A}_{*} \otimes C_{*}(K)$ and $\boldsymbol{A}_{*}^{K}$ by $\boldsymbol{A}_{*}^{C_{*}(K)}$; hence, instead of $K$, the associated chain complexes appear. So this works for simplicial sets as well as for simplicial spectra $K$. By this procedure, a tensor product

$$
-\otimes-: \mathfrak{L} \otimes \mathfrak{S p} \longrightarrow \mathfrak{L}
$$

and a cotensor product

$$
()^{(~)}: \mathfrak{L} \otimes \mathfrak{S p}^{o p} \longrightarrow \mathfrak{L}
$$

are established, together with some $\operatorname{Hom}\left(L_{1}, L_{2}\right) \in \mathfrak{S p}, L_{1}, L_{2} \in \mathfrak{L}$, satisfying the modified properties SM6 and SM7 in $\S 11$. In order to be able to accomplish this, we need that $\mathfrak{C h}$ is a stable model category (i.e. the suspension is invertible on the homotopy level). By checking this we realize that we will lose the associativity (even up to weak equivalences) of the tensor product (Lemma 2.7 for the tensor product with $\mathfrak{S})$, because we can not find the analog of EZ-mappings in our case; they are established by an inductive process, which unlike simplicial sets, is not available for spectra.

In the same way as for simplicial sets, we associate with each $X \in \mathfrak{S p}$ a chain complex $C_{*}(X) \in \mathfrak{c h}$ of free abelian groups, which for each degree $q$ is generated by the simplexes of degree $q$, with boundary

$$
d \sigma^{q}=\sum_{i=0}^{\infty}(-1)^{i} \partial_{i} \sigma^{q}
$$

which is, according to the definition of simplicial spectra, in fact a finite sum.

A cofibration in $\mathfrak{S p}$ is an inclusion. A fibration $p: X \longrightarrow Y$ is defined in complete analogy to a Kan fibration in $\mathfrak{S}$ by the requirement that a given horn $\Lambda_{k}^{q-1}$ in $X$ has a filling, which is mapped under $p$ into a given filling of the image of this horn $p\left(\Lambda_{k}^{q-1}\right)$ in $Y$. Instead of defining weak equivalences in $\mathfrak{S p}$ directly, we simply take all those $f \in \mathfrak{S p}(X, Y)$ for which $C_{*}(f)$ becomes a chain homotopy equivalence in $\mathfrak{c h}$. We do not claim (nor do we know) whether this defines a model structure in $\mathfrak{S p}$. The authors did not find any reference to the existence of a model structure for $\mathfrak{S p}$ specifically implementing these classes of morphisms. All these definitions are sufficient to establish Proposition 2.5 for simplicial spectra instead of simplicial sets:

Proposition 7.2. 1. Suppose that $j: K \subset L$ is a (trivial) cofibration in $\mathfrak{S p}$ and that $\boldsymbol{A}_{*} \in \mathfrak{C h}$. Then $\boldsymbol{A}_{*} \otimes j$ is a (trivial) cofibration in $\mathfrak{C h}$.

2. Let $q: \boldsymbol{A}_{*} \subset \boldsymbol{B}_{*}$ be a (trivial) cofibration in $\mathfrak{C h}, X \in \mathfrak{S p}$. Then $q \otimes X$ is a (trivial) cofibration in $\mathfrak{C h}$.

In the same manner, we note that all results of $\S 3$ can be immediately formulated with $\mathfrak{S p}$ replacing $\mathfrak{S}$. In particular, we have the following kind of adjointness, 
deduced from (5) of $\S 3$ :

$$
\mathfrak{C h}\left(\boldsymbol{A}_{*} \otimes X, \boldsymbol{B}_{*}\right) \approx \mathfrak{C h} \mathfrak{h}\left(\boldsymbol{A}_{*}, \boldsymbol{B}_{*}^{X}\right), X \in \mathfrak{S p}, \boldsymbol{A}_{*}, \boldsymbol{B}_{*} \in \mathfrak{C h},
$$

where

$$
()^{(~)}: \mathfrak{C h} \otimes \mathfrak{S p}^{o p} \longrightarrow \mathfrak{C h} \mathfrak{h}
$$

is the cotensor functor from $§ 3$. Proposition 3.7 carries over immediately.

We come to property SM7 for the tensor product and note that the proof of Theorem 5.3 immediately yields:

Theorem 7.3. The tensor product

$$
-\otimes-: \mathfrak{C h} \times \mathfrak{S p} \longrightarrow \mathfrak{C h}
$$

described above satisfies SM\%:

Let $q: \boldsymbol{A}_{*} \subset \boldsymbol{B}_{*}$ be a cofibration in $\mathfrak{C h}$ and $j: X \subset Y$ be a cofibration in $\mathfrak{S p}$. Then there exists a pushout $\boldsymbol{P}_{*}=\boldsymbol{A}_{*} \cup_{\boldsymbol{A}_{*} \otimes X} \boldsymbol{B}_{*} \otimes Y$ in $\mathfrak{C h}$ and a mapping

$$
w=\left(\boldsymbol{B}_{*} \otimes j, q \otimes Y\right): \boldsymbol{P}_{*} \longrightarrow \boldsymbol{B}_{*} \otimes Y
$$

which is a cofibration. Moreover $w$ is a trivial cofibration whenever either $q$ or $j$ is a trivial cofibration.

Suppose $\boldsymbol{A}_{*}, \boldsymbol{B}_{*} \in \mathfrak{C h}$. Then we define a simplicial spectrum $\operatorname{Hom}\left(\boldsymbol{A}_{*}, \boldsymbol{B}_{*}\right)$ in the following way: Let $K_{*} \in \mathfrak{c h}$. Then we define a suspension $\Sigma^{t} K_{*}$ by

$$
\left(\Sigma^{t} K\right)_{n+t}=K_{n}, t \in \mathbb{Z} \text {. }
$$

Now we set

$$
\operatorname{Hom}\left(\boldsymbol{A}_{*}, \boldsymbol{B}_{*}\right)_{t}=\bigcup_{n=0}^{\infty} \mathfrak{C h}\left(\boldsymbol{A}_{*} \otimes \Sigma^{t} \Delta^{n}, \boldsymbol{B}_{*}\right) / \sim, t \in \mathbb{Z}
$$

where the equivalence relation is defined by identifying $\Delta^{n-1}$ with $\partial_{n} \Delta^{n}$. Boundaries and degeneracies are induced by those of $\Delta^{n}$. It is obvious that $\operatorname{Hom}\left(\boldsymbol{A}_{*}, \boldsymbol{B}_{*}\right)$ is an object of $\mathfrak{S p}$ and that

$$
\text { Hom : } \mathfrak{C h}^{o p} \times \mathfrak{C h} \longrightarrow \mathfrak{S p}
$$

is a functor.

We can now transfer all results from $\S 4$; in particular the adjointness

$$
\mathfrak{S p}\left(X, \operatorname{Hom}\left(\boldsymbol{A}_{*}, \boldsymbol{B}_{*}\right)\right) \approx \mathfrak{C h}\left(\boldsymbol{A}_{*} \otimes X, \boldsymbol{B}_{*}\right),
$$

and Theorem 4.2, Lemma 4.4 to our present case. As a result we obtain SM7 for the Hom-functor:

Theorem 7.4. Let $q: \boldsymbol{A}_{*} \subset \boldsymbol{B}_{*}$ be a cofibration and $p: \boldsymbol{X}_{*} \longrightarrow \boldsymbol{Y}_{*}$ a regular fibration in $\mathfrak{C h}, P \in \mathfrak{S p}$ the pullback as in $\S 6(1), w=\left(q^{*}, p_{*}\right) \in \mathfrak{S p}\left(\boldsymbol{H o m}\left(\boldsymbol{B}_{*}, \boldsymbol{X}_{*}\right), P\right)$. Then $w$ is a fibration in $\mathbf{S p}$, which is trivial, if either $q$ is a trivial cofibration or $p$ a trivial fibration.

Remark 7.5. 1) Analogously to what we did in Lemma 2.8, we can now define the suspension of a chain functor $\boldsymbol{A}_{*}$ by $\boldsymbol{A}_{*} \otimes \Sigma S^{0}$, where $S^{0}$ denotes the sphere spectrum ([10], §2, Example 2.2). 
Homology, Homotopy and Applications, vol. 9(1), 2007

2) The advantage of the category $\mathfrak{S p}$ is the fact that the verification of all properties of a ss-model structure is not much more than a repetition of the related proofs for $\mathfrak{S}$ in the previous sections. The disadvantage of the choice of $\mathfrak{S p}$ is embodied in the absence of some pleasant properties of this category in comparison with the categories of spectra mentioned at the beginning of this section.

\section{8. $\mathfrak{C h}$ and the proper model category structure}

In this section we verify that $\mathfrak{C h}$ satisfies the condition of a (left and right) proper model structure.

Let $\mathfrak{K}$ be any category satisfying all conditions of a model category, with the possible exception of CM1.

$(\mathrm{LP}) \mathfrak{K}$ is called left proper whenever the following holds: Let

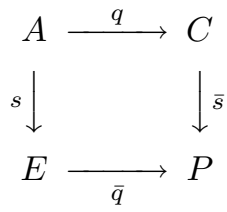

be a pushout diagram, $q$ a cofibration, $s$ a weak equivalence. Then $\bar{s}$ is a weak equivalence.

$(\mathrm{RP}) \mathfrak{K}$ is right proper whenever the following holds: Let

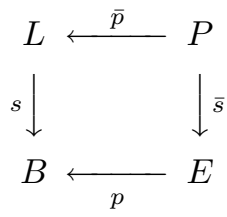

be a pullback diagram, $p$ a fibration and $s$ a weak equivalence. Then $\bar{s}$ is a weak equivalence.

(P) A category $\mathfrak{K}$ is proper if it is left and right proper.

Concerning this definition and its applications in the general theory of model structures, we refer to [7].

We are going to prove:

Theorem 8.1. The category $\mathfrak{C h}$ is proper. More precisely, whenever the pushout (1) (resp. pullback (2)) exists, (LP) (resp. (RP)) holds.

For this and the following proofs we only need some intrinsic properties of the category $\mathfrak{C h}$, and not specific properties of chain functors, so we denote the objects simply by $A, B, C, \ldots$ rather than by $\boldsymbol{A}_{*}, \ldots$.

A mapping $q: A \longrightarrow C$ is a cofibration whenever a homotopy $\omega: f \simeq g: A \longrightarrow$ $X, f=f^{\prime} q, f^{\prime}: C \longrightarrow X$, determines a homotopy $\omega^{\prime}: f^{\prime} \simeq g^{\prime}$ such that $\omega^{\prime} q=\omega$ and therefore $g^{\prime} q=g$.

We concentrate our efforts on a proof of: 
Proposition 8.2. The category $\mathfrak{C h}$ is left proper.

We need:

Lemma 8.3. There exists a $\bar{t}: P \longrightarrow C$ such that $\bar{t} \bar{s} \simeq 1$.

Proof. Since $s$ is a weak equivalence in $\mathfrak{C h}$, hence a homotopy equivalence, we have a $t: E \longrightarrow A$ and a homotopy $\omega_{1}: t s \simeq 1$. So we have $\omega=q \omega_{1}: q t s \simeq q$. Since $q$ is a cofibration, we detect an $a: C \longrightarrow C$ and a homotopy $\omega^{\prime}: a \simeq 1$, such that $\omega^{\prime} q=\omega, a q=q t s$. Since $(1)$ is a pushout diagram, there exists a $\bar{t}: P \longrightarrow C$ such that $\bar{t} \bar{q}=q t, \bar{t} \bar{s}=a$. This implies $\bar{t} \bar{s} \simeq 1$.

It turns out to be helpful for the proof of Lemma 8.5 as well of independent interest to include the following assertion, together with a proof in our present terminology (cf. 2.6):

Proposition 8.4. 1) Let (1) be a pushout diagram in $\mathfrak{C h}, q$ a cofibration. Then $\bar{q}$ is a cofibration.

2) Let (2) be a pullback diagram and $p$ be a fibration. Then $\bar{p}$ is a fibration.

Proof. We deal with the first assertion: Suppose $\omega: f \simeq g, g=g^{\prime} \bar{q}: E \longrightarrow X$. Then, because $q$ is a cofibration, we find a homotopy $\nu: g^{\prime} \bar{s} \simeq h$ such that $\nu q=$ $\omega s, q h=f s$. Now we apply the fact that in the category $\mathfrak{C h}$, a homotopy $\gamma: f_{0} \simeq$ $f_{1}: X \longrightarrow Y$ can be expressed by a mapping $\gamma=H_{\gamma}: X \longrightarrow Y^{I}$ such that $p_{i} H_{\gamma}=$ $f_{i}, i=0,1$ (cf. [4], Proposition 1.4). So for $H_{\omega}: E \longrightarrow X^{I}$ and $H_{\nu}: C \longrightarrow X^{I}$ there exists, by the pushout property of (1), a unique $\omega^{\prime}: g^{\prime} \simeq h^{\prime}$ satisfying $\omega^{\prime} \bar{q}=\omega$ and $h^{\prime} \bar{q}=f$. This confirms that $\bar{q}$ is a cofibration. The assertion about pullbacks and fibrations is entirely dual.

Lemma 8.5. There exists a homotopy $\bar{s} \bar{t} \simeq 1$; hence $\bar{s}$ is a weak equivalence in $\mathfrak{C h}$.

Proof. According to Proposition 8.4, we detect a $b: P \longrightarrow P$ and a homotopy $\nu: \bar{s} \bar{t} \simeq b$ such that $b \bar{q}=\bar{q}$, yielding a homotopy $\alpha: E \longrightarrow P^{I}$ satisfying

$$
\alpha_{0}=p_{0} \alpha=\bar{s} \bar{t} \bar{q}, \alpha_{1}=p_{1} \alpha=1_{P} \bar{q} .
$$

There also exists a homotopy $b \bar{s} \simeq \bar{s} \bar{s} \simeq \bar{s}$ (using 8.3), hence a homotopy $\beta: C \longrightarrow$ $P^{I}$, satisfying

$$
\begin{gathered}
\beta_{0}=\bar{s} \bar{t} \bar{s}, \beta_{1}=1_{P} \bar{s}, \\
\beta q=\alpha s .
\end{gathered}
$$

Since (1) is a pushout diagram, there exists a unique (right) homotopy $\gamma: P \longrightarrow P^{I}$ satisfying

$$
\gamma \bar{q}=\alpha, \gamma \bar{s}=\beta
$$

The uniqueness property of $\gamma$ implies

$$
\gamma_{0}=\bar{s} \bar{t}, \gamma_{1}=1_{P}
$$


Proof of 8.2. Follows immediately from 8.3 and 8.5.

Proof of 8.1. All conclusions in the preceding proofs can immediately be dualized (replacing cofibrations by fibrations, pushouts by pullbacks and right homotopies by left homotopies $X \times I \longrightarrow Y$ ).

\section{Proof of Proposition 1.1}

For this purpose we need the definition of a chain functor, hence the properties $\mathrm{CH} 1-\mathrm{CH} 7$ which are recorded e.g. in [3], $\S 7$ or in [4], $\S 9$ and in $\S 10$ of the present paper. In addition we need the following:

Lemma 9.1. Let $\boldsymbol{A}_{*}$ be any chain functor, $d c=k z, c \in A_{*}(X, U), k \in \mathbb{Z}$. Then there exist $z^{\prime}, c^{\prime} \in A_{*}^{\prime}(X, U), u_{i} \in A_{*}(U, U), i=1,2, u_{3} \in A_{*}(U)$ such that

$$
\begin{gathered}
l z^{\prime}+q_{\#} u_{1} \sim z, d z^{\prime} \in i m i^{\prime} \\
l c^{\prime}+q_{\#} u_{2}=c+d w, w \in A_{*}(X, U) \\
k z^{\prime}+i^{\prime} u_{3}=d c^{\prime} .
\end{gathered}
$$

Proof. This is [2], Lemma 1.1.

The proof of Proposition 1.1, which we are going to display in detail, consists mainly of an analysis of the proof of the Künneth formula

$$
\begin{array}{r}
0 \rightarrow\left(H_{*}\left(A_{*}(X, U)\right) \otimes H_{*}\left(K_{*}\right)\right)_{n} \rightarrow H_{n}\left(A_{*} \otimes K_{*}\right)(X, U) \\
\rightarrow\left(H_{*}\left(A_{*}(X, U)\right) \star H_{*}\left(K_{*}\right)\right)_{n-1} \rightarrow 0 .
\end{array}
$$

The defining properties of a chain functor are, for the reader's convenience, recorded in $\S 10$.

Proof of Proposition 1.1. Observe that $A_{*}(X, U)$, as well as $K_{*}$, are free chain complexes, so that (1) holds. For this we need only that $H\left(A_{*} \star K_{*}\right)=0$ (cf. [5], Ch. VI).

Property $\mathrm{CH} 1$ provides the ingredients of a chain functor like $\left(A_{*} \otimes K_{*}\right)_{*}^{\prime} l, i^{\prime}$, $\varphi, \kappa$. This was already accomplished in $\S 1$. Property $\mathrm{CH} 2$ states that all inclusions induce monomorphisms and that $H_{*}\left(A_{*} \otimes K_{*}\right)(X, X)=0$. This has been already accomplished in $\S 1$ or follows immediately from (1). Let $p:(X, U) \longrightarrow(Y, V)$ be an excision map. Then $p$ induces an isomorphism in homology for $A_{*}$; hence, because (1) is natural, an isomorphism for $B_{*}(p)=A_{*}(p) \otimes K_{*}$. This confirms property CH6.

Since $A_{*}(X, U)$ as well as $K_{*}$ is free, the former with a natural basis, we conclude that $B_{*}(X, U)=A_{*}(X, U) \otimes K_{*}$ is also free, with a natural basis. This confirms $\mathrm{CH}$ 7. Property $\mathrm{CH} 5$ for $B_{*}$ follows immediately from the same property for $A_{*}$.

We now have to verify $\mathrm{CH} 3$ and $\mathrm{CH} 4$; what are cycles $z \in Z_{n}\left(B_{*}(X, U)\right)$ ? According to (1), we can assume that they are sums of two different types of cycles:

ऽ 1) $\hat{z}=z_{A} \otimes z_{K}, d z_{A}=0, d z_{K}=0$

$\zeta 2) \tilde{z}=z_{A} \otimes b+(-1)^{p+1} a \otimes z_{K}, \operatorname{dim} z_{A}=p$, where for some $m \in \mathbb{Z}$ we have $d a=m z_{A}, d b=m z_{K}$. 
The first one appears as a special case of the second one by setting $d a=0, z_{A}=0$.

Let $\hat{z}$ be a cycle and assume that there exists a $m \in \mathbb{Z}$ and a $b \in K_{*}$ such that $d b=m z_{k}$. Then $\hat{z}$ is bounding, whenever there exist $t_{1}, t_{2} \in A_{*}$ such that

$$
\hat{z}=d\left(t_{1} \otimes z_{K}+t_{2} \otimes b\right),
$$

hence, $d t_{2}=0, z_{A}=d t_{1}+(-1)^{p} m t_{2}$.

If a cycle $\tilde{t}$ of the form $\zeta 2$ ) is bounding. Then

$$
z_{A} \otimes b+(-1)^{p+1} a \otimes z_{K}=d\left(x \otimes b+(-1)^{p+1} t \otimes z_{K}\right) ;
$$

hence $d x=z_{A}, a-m x=d t$.

$\underline{\text { CH3 }}$ : Let $\hat{z}=z_{A} \otimes z_{K}, z_{A} \sim l z_{A}^{\prime}+q_{\#} u_{1}$. Then $\hat{z} \sim l\left(z_{A}^{\prime} \otimes z_{K}\right)+q_{\#} u_{1} \otimes z_{K}, z_{A}^{\prime} \in$ $A_{*}^{\prime}(X, U), u_{1} \in A_{*}(U, U)$.

Let $\tilde{z}=z_{A} \otimes b+(-1)^{p+1} a \otimes z_{K}$ be a cycle, with $z_{A} \sim l z_{A}^{\prime}+q_{\#} u_{1}$ and $a=l a^{\prime}+$ $q_{\#} u_{2}+d w$ as in Lemma 9.1. Then

$$
\begin{gathered}
\tilde{z} \sim l z_{A}^{\prime} \otimes b+(-1)^{p+1} l a^{\prime} \otimes z_{K}+q_{\#}\left(u_{1} \otimes b+(-1)^{p+1} u_{2} \otimes z_{K}\right) \\
=l z^{\prime}+q_{\#} \tilde{u}, d z^{\prime} \in i m i^{\prime} .
\end{gathered}
$$

$\underline{\mathrm{CH} 4}:$

$$
\text { ker } \psi \subset \operatorname{ker} \bar{\partial} \text {. }
$$

Assume $\hat{z}=z_{A} \otimes z_{K}=d\left(t_{1} \otimes z_{K}+t_{2} \otimes b\right)$. Then we have $d t_{2}=0, d b=m z_{K}, \hat{z}=$ $l z_{A}^{\prime} \otimes z_{K}+q_{\#}\left(u_{1} \otimes z_{K}\right), z_{A}=d t_{1}+(-1)^{p} m t_{2}$, hence $d t_{1}=l\left(z_{A}^{\prime}+(-1)^{p+1} m t_{2}^{\prime}\right)$ $+q_{\#}(-1)^{p+1} m u_{2}+u_{1}$ and, due to CH4 for $\boldsymbol{A}_{*}$ :

$$
\begin{gathered}
d\left(z_{A}^{\prime}+(-1)^{p+1} m t_{2}^{\prime}\right)=d u, u \in A_{*}(U) . \\
d\left(z_{A}^{\prime} \otimes z_{K}\right)=(-1)^{p} m d t_{2}^{\prime} \otimes z_{K}+d\left(u \otimes z_{K}\right) \in B_{*}(U) .
\end{gathered}
$$

This confirms (2) for $\hat{z}$.

Suppose $\tilde{z}=z_{A} \otimes b+(-1)^{p+1} a \otimes z_{K}=d\left(x \otimes z_{K}+(-1)^{p+1} t \otimes b\right)=l \tilde{z}^{\prime}+q_{\#} \tilde{u}$. We know already that $z_{A}=d x, a-m x=d t$.

We have:

$$
\begin{gathered}
\tilde{z}=z_{A} \otimes b+(-1)^{p+1} m x \otimes z_{K}+(-1)^{p+1}(a-m x) \otimes z_{K} \\
=\tilde{z}_{1}+\hat{z}_{2}=l \tilde{z}_{1}^{\prime}+q_{\#} \tilde{u}_{1}+l \tilde{z}_{2}^{\prime}+q_{\#} \tilde{u}_{2},
\end{gathered}
$$

and calculate:

$$
d \tilde{z}_{1}^{\prime}=d z_{A}^{\prime} \otimes b+(-1)^{p}\left(m z_{A}^{\prime}-m d x^{\prime}\right) \otimes z_{K},
$$

$x=l x^{\prime}+q_{\#} u_{2}+d w$. Due to CH4 for $\boldsymbol{A}_{*}$ and because $m z_{A}$ is bounding, we conclude that

$$
z_{A}=l z_{A}^{\prime}+q_{\#} d u, u \in A_{*}(U) .
$$

Hence

$$
d \tilde{z}_{1}^{\prime}=-d u \otimes b+(-1)^{p+1} m u \otimes z_{K}+(-1)^{p} d\left(m u_{2}\right) \otimes z_{K}
$$


Homology, Homotopy and Applications, vol. 9(1), 2007

$$
=-d\left(u \otimes b-d u_{2} \otimes b\right) .
$$

Since $u \otimes b-d u_{2} \otimes b \in B_{*}(U)$, this yields (2) for $\tilde{z}_{1}$. The case of $\hat{z}_{2}$ has already been settled earlier. This confirms (2) for $\tilde{z}$.

We come to the verification of

$$
\operatorname{ker} j_{*} \subset \operatorname{ker} p_{*} \kappa_{*}
$$

for $\boldsymbol{B}_{*}$. Firstly we observe that this is equivalent to the following formulation: Let $\boldsymbol{A}_{*}$ be any chain functor, $z \in A_{*}(X, U)$ a cycle, satisfying $j_{\#} z \sim 0$ in $A_{*}(X, U)$. Then there exists $u \in A_{*}(U), x \in A_{*}(X)$ such that

$$
z=i_{\#} u+d x .
$$

The original formulation of (3) ensures the existence of a $u \in A_{*}(U)$, as well of $x^{\prime} \in A_{*}^{\prime}(X, U)$, such that

$$
\kappa z=i^{\prime} u+d x^{\prime} .
$$

Application of $\varphi$ to (5) yields (4). In the same way, we use $i^{\prime}=\kappa i_{\#}$ to deduce (5) from (4).

We merely have to deal with $\tilde{z}=z_{A} \otimes b+(-1)^{p+1} a \otimes z_{K}$; the case $\hat{z}=z_{A} \otimes z_{K}$ is included in the first one by setting $z_{A}=0, d a=0$.

Recall that $j_{\#} z_{A}=d x, j_{\#} a-m x=d t, d a=m z_{A}, d b=m z_{k}$.

First of all we establish a representation

$$
x=l x^{\prime}+q_{\#} v+d s, v \in A_{*}(U, U), d v \in A_{*}(U), x^{\prime} \in A_{*}^{\prime}(X, U)
$$

in the following way: Since $d x=j_{\#} z_{A}$, we have, due to CH4 for $\boldsymbol{A}_{*}$,

$$
z_{A}=i_{\#} w+d y, w \in A_{*}(U), y \in A_{*}(X) ;
$$

hence

$$
d x=j_{\#} i_{\#} w+d j_{\#} y,
$$

implying that $c=x-\left(q_{\#} \bar{w}-j_{\#} y\right), d \bar{w}=w, \bar{w} \in A_{*}(U, U)$ is a cycle. By CH1, $c=$ $l c^{\prime}+q_{\#} w_{1}+d s_{1}, d w_{1} \in A_{*}(U), w_{1} \in A_{*}(U, U), s_{1} \in A_{*}(X, U)$. As a result

$$
x=l c^{\prime}+j_{\#} y+q_{\#} \bar{w}+d s_{2},
$$

but there exists an $x^{\prime} \in A^{\prime}(X, U)$ such that $l x^{\prime}=l c^{\prime}+j_{\#} y$, confirming (6).

Now we calculate:

$$
\begin{gathered}
j_{\#} z_{A}=d l x^{\prime}+d v, \\
j_{\#} a=m l x^{\prime}+m q_{\#} v+d(t+m s), \\
j_{\#}\left(a-m \varphi x^{\prime}\right)=m q_{\#} v+d t_{1}, t_{1} \in A_{*}(X, U) .
\end{gathered}
$$

We interpret $j_{\#}\left(a-m \varphi x^{\prime}\right)$ as $\left(j_{\#}\left(a-m \varphi x^{\prime}\right)-m q_{\#} v\right)^{\prime}$ and deduce from CH4 (for $\boldsymbol{A}_{*}$ ) that $m v=d \bar{v}, \bar{v} \in A_{*}(U)$. 
Now we again apply $\mathrm{CH} 4$ to the result that

$$
a-m \varphi x^{\prime}=v_{2}+d y_{2}, v_{2} \in A_{*}(U), y_{2} \in A_{*}(X) .
$$

Now (7) implies

$$
j_{\#}\left(d y-d \varphi x^{\prime}-i_{\#} d v\right)=0
$$

hence

$$
d y=d \varphi x^{\prime}+i_{\#} w_{3}, w_{3} \in A_{*}(U) .
$$

Now we calculate:

$$
\begin{aligned}
z_{A} \otimes & b+(-1)^{p+1} a \otimes z_{K} \\
= & i_{\#} w \otimes b+(-1)^{p+1} i_{\#} v_{2} \otimes z_{K} \\
& +\left[d y \otimes b+(-1)^{p+1} m \varphi x^{\prime} \otimes z_{K}+(-1)^{p+1} d y_{2} \otimes z_{K}\right] \\
= & i_{\#}\left(w+w_{3}\right) \otimes b+i_{\#}(-1)^{p+1} v_{2} \otimes z_{K} \\
& +d \varphi x^{\prime} \otimes b+(-1)^{p+1} m \varphi x^{\prime} \otimes z_{K}+d\left(y_{2} \otimes z_{K}\right) \\
= & i_{\#}\left(\tilde{w} \otimes b+(-1)^{p+1} v_{2} \otimes z_{K}\right)+d\left(\varphi x^{\prime} \otimes b+y_{2} \otimes z_{K}\right) .
\end{aligned}
$$

This completes the proof of $\mathrm{CH} 4$ and therefore of Proposition 1.1.

We have the following immediate corollary of Proposition 1.1:

Corollary 9.2. The construction of the tensor product in $\S 1$ leads to a functor

$$
-\otimes-: \mathfrak{C h} \mathfrak{h} \times \mathfrak{c h} \longrightarrow \mathfrak{C h} h .
$$

Proposition 9.3. The assignment

$$
\boldsymbol{A}_{*} \longmapsto \boldsymbol{A}_{*}^{K_{*}}, \boldsymbol{A}_{*} \in \mathfrak{C h} \text { or } \boldsymbol{A}_{*} \in \mathfrak{C h} \mathfrak{h}
$$

yields a functor

$$
\mathfrak{C h h} \times \mathfrak{S}^{o p} \longrightarrow \mathfrak{C h} \mathfrak{h}
$$

Proof. Here we apply the $* \backslash$-convention of A. Dold (cf. [5], Ch. VI, §2), replacing the Künneth formula (1) by the corresponding Künneth formula for function complexes ([5], Theorem 10.11)

$$
\begin{aligned}
0 \rightarrow \operatorname{Ext}\left(H_{*}\left(K_{*}\right)\right. & \left., H_{*}\left(A_{*}(X, U)\right)\right)_{n+1} \rightarrow H_{n}\left(A_{*}^{K_{*}}(X, U)\right) \\
& \rightarrow \operatorname{Hom}\left(H_{*}\left(K_{*}\right), H_{*}\left(A_{*}(X, U)\right)_{n} \rightarrow 0,\right.
\end{aligned}
$$

and proceed with the translation of the proof of Proposition 1.1 according to A. Dold's $* \backslash$ convention. Details are left to the reader.

\section{The definition and motivation of a chain functor}

For the reader's convenience and to make the present paper independent, we include here the definition of a chain functor based on [3], §7. This will be essential for the detailed verification of the chain functor properties in $\S 9$. We refer to [1] or [2] for further details about chain functors. 
It would be advantageous to define a homology theory $h_{*}($ ) as the derived homology of a functor

$$
C_{*}: \mathfrak{K} \longrightarrow \mathfrak{c h},
$$

where $\mathfrak{K}$ is the category on which $h_{*}$ is defined. For us this will be always either a subcategory of the category of all pairs of topological spaces, or of pairs of spectra or of pairs of $\mathrm{CW}$ spaces, of $\mathrm{CW}$ spectra, or their simplicial counterparts. ch denotes the category of chain complexes of free abelian groups and $\mathfrak{c h} \mathfrak{h}$ denotes the category of not necessarily free chain complexes.

Let $(X, U) \in \mathfrak{K}$ be a pair. Then one would like to have an exact sequence (writing $C_{*}(X)$ instead of $\left.C_{*}(X, \emptyset)\right)$

$$
0 \longrightarrow C_{*}(U) \stackrel{i_{\#}}{\longrightarrow} C_{*}(X) \stackrel{j_{\#}}{\longrightarrow} C_{*}(X, U) \longrightarrow 0
$$

such that the associated boundary $\bar{\partial}: H_{n}\left(C_{*}(X, U)\right) \longrightarrow H_{n-1}\left(C_{*}(U)\right)$ induces the boundary $\partial: h_{n}(X, U) \longrightarrow h_{n-1}(U)$ of the homology theory $h_{*}()$.

We call a homology theory $h_{*}() \approx H_{*}\left(C_{*}()\right)$, where $C_{*}()$ is of this sort, flat. Due to a result of R. O. Burdick, P. E. Conner and E. E. Floyd (see [1] for further reference) this implies, for $\mathfrak{K}$ the category of $\mathrm{CW}$ pairs, that $h_{*}()$ is a sum of ordinary homology theories, i.e. of those satisfying a dimension axiom, although not necessarily in dimension 0 . We call a functor $C_{*}$ equipped with a short exact sequence (1) and determining the boundary operator a chain theory for $h_{*}$. The non-existence of such a chain theory gives rise to the theory of chain functors.

A chain functor $C_{*}=\left\{C_{*}, C_{*}^{\prime}, l, i^{\prime}, \kappa, \varphi\right\}$ is a pair of functors $C_{*}, C_{*}^{\prime}: \mathfrak{K} \longrightarrow \mathfrak{c h}$, natural inclusions $i^{\prime}: C_{*}(U) \subset C_{*}^{\prime}(X, U), l: C_{*}^{\prime}(X, U) \subset C_{*}(X, U)$, non-natural chain mappings

$$
\begin{gathered}
\varphi: C_{*}^{\prime}(X, U) \longrightarrow C_{*}(X) \\
\kappa: C_{*}(X) \longrightarrow C_{*}^{\prime}(X, U),
\end{gathered}
$$

satisfying conditions $\mathbf{C H 1}-\mathbf{C H 7}$ below:

CH1: There exist (of course in general non-natural) chain homotopies $\varphi \kappa \simeq 1$, $j_{\#} \varphi \simeq l \quad(j: X \subset(X, U))$, as well as an identity

$$
\kappa i_{\#}=i^{\prime}, \quad i: U \subset X .
$$

CH2: All inclusions $k:(X, U) \subset(Y, V)$ induce monomorphisms on $C_{*}$. Each $C_{*}(X, X)$ is acyclic.

It should be observed that the chain complexes $C_{*}(X, U)$ appearing in (1) are not identical to the chain complexes $C_{*}(X, U)$ appearing in a chain functor. The latter have the property that for all pairs $(X, U)$, one has inclusions $C_{*}(X)=C_{*}(X, \emptyset) \subset$ $C_{*}(X, U) \subset C_{*}(X, X)$. These groups cannot be members of a short exact sequence (1).

Needless to say, $C_{*}^{\prime}$, as well as $\phi, \kappa$, are not determined by the functor $C_{*}(-,-)$ but are additional ingredients of the structure of a chain functor.

Instead of the exact sequence (1) for chain theories we are now, in the case of a 
chain functor, dealing with the sequence

$$
0 \longrightarrow C_{*}(U) \stackrel{i^{\prime}}{\longrightarrow} C_{*}^{\prime}(X, U) \stackrel{p}{\longrightarrow} C_{*}^{\prime}(X, U) / i m i^{\prime} \longrightarrow 0
$$

and there exists a homomorphism

$$
\begin{gathered}
\psi: H_{*}\left(C_{*}^{\prime}(X, U) / i m i^{\prime}\right) \longrightarrow H_{*}\left(C_{*}(X, U)\right) \\
{\left[z^{\prime}\right] \quad \longmapsto\left[l\left(z^{\prime}\right)+q_{\#}(\bar{a})\right]}
\end{gathered}
$$

where $z^{\prime} \in C_{*}^{\prime}(X, U), d z^{\prime} \in i m i^{\prime}, q:(A, A) \subset(X, U), \bar{a} \in C_{*}(U, U), d \bar{a}=-d z^{\prime}$. Вy this assignment, $\psi$ is readily defined.

CH3: It is assumed that $\psi$ is epic.

Since $C_{*}(U, U)$ is acyclic and $d z^{\prime} \in i m i^{\prime}$, there exists an $\bar{a}$ with $q_{\#}(\bar{a})=-d l\left(z^{\prime}\right)$ and $\left[l\left(z^{\prime}\right)+q_{\#}(\bar{a})\right]$ turns out to be independent of the choice of $\bar{a}$.

This assumption implies that whenever (1) holds, i.e. whenever we are dealing with a chain theory, each cycle $z \in C_{*}(X, U)$ is homologous to a cycle of the form $l\left(z^{\prime}\right)+q_{\#}(\bar{a})$, with $z^{\prime}$ being a relative cycle, the analogue of a classical relative cycle $z \in C_{*}(X)$ with $d z \in i m i_{\#}$.

Suppose $\bar{\partial}: H_{n}\left(C_{*}^{\prime}(X, U) / i m i^{\prime}\right) \longrightarrow H_{n-1}\left(C_{*}(U)\right)$ is the boundary induced by the exact sequence $(2)$.

CH4: We assume

$$
\text { ker } \psi \subset k e r \bar{\partial} \text {. }
$$

Moreover

$$
\text { ker } j_{*} \subset \operatorname{ker} p_{*} \kappa_{*}
$$

with e.g. $\kappa_{*}$ denoting the mapping induced by $\kappa$ for the homology groups.

CH5: Homotopies $H:(X, U) \times I \longrightarrow(Y, V)$ naturally induce chain homotopies $D(H): C_{*}(X, U) \longrightarrow C_{*+1}(Y, V)$ that are compatible with $i^{\prime}$ and $l$.

The derived (or associated) homology of a chain functor

$$
h_{*}(X, U)=H_{*}\left(C_{*}(X, U)\right)
$$

(resp. for the induced mappings), has a boundary operator $\partial: H_{n}\left(C_{*}(X, U)\right) \longrightarrow$ $H_{n-1}\left(C_{*}(U)\right)$ determined by $\bar{\partial}$ : Given $\zeta \in H_{n}\left(C_{*}(X, U)\right)$ we choose a lift $z^{\prime}$, which exists by $\mathrm{CH} 3$, a representative $l\left(z^{\prime}\right)+q_{\#}(\bar{a}) \in \zeta$ and set

$$
\partial \zeta=\bar{\partial}\left[z^{\prime}\right]=\left[i^{\prime-1} d z^{\prime}\right]
$$

This turns out to be independent of the choices involved.

This $h_{*}($ ) satisfies all properties of a homology theory with the possible exception of an excision. Let us assume that in $\mathfrak{K}^{2}$ there are some mappings $p:(X, U) \longrightarrow$ $\left(X^{\prime}, U^{\prime}\right)$ serving as excision maps (of some kind, e.g. $p:(X, U) \longrightarrow(X / U, \star)$ ). Then it is convenient to add:

CH6: Let $p$ be an excision map. Then $p_{*}=H_{*}\left(C_{*}(p)\right)$ is required to be an isomorphism. 
This $H_{*}\left(C_{*}()\right)=h_{*}()$ turns out to be a homology theory. Moreover, under very general conditions on $\mathfrak{K}$, every homology theory $h_{*}()$ is isomorphic to the derived homology of some chain functor (see [1] for further references).

Let $\lambda: C_{*} \longrightarrow L_{*}, \lambda^{\prime}: C_{*}^{\prime} \longrightarrow L_{*}^{\prime}$ be natural transformations, where $\boldsymbol{C}_{*}, \boldsymbol{L}_{*}$ are chain functors compatible with $i^{\prime}, l$ and the natural homotopies of CH5. Then we call $\lambda: \boldsymbol{C}_{*} \longrightarrow \boldsymbol{L}_{*}$ a transformation of chain functors. Such a transformation induces obviously a transformation $\lambda_{*}: H_{*}\left(\boldsymbol{C}_{*}\right) \longrightarrow H_{*}\left(\boldsymbol{L}_{*}\right)$ of the derived homology. This furnishes a category $\mathfrak{C h}$ of chain functors. A weak equivalence in $\mathfrak{C h}$ is a $\lambda: \boldsymbol{C}_{*} \longrightarrow \boldsymbol{L}_{*}$ which has a homotopy inverse.

Furthermore we can introduce the homotopy category $\mathfrak{C h}_{h}$ with chain homotopy classes of transformations of chain functors as morphisms (alternatively, $\mathfrak{C h}_{h}=$ $\mathfrak{C h} /\{\mathfrak{W}\}, \mathfrak{W}=$ class of weak equivalences, i.e. all weak equivalences in $\mathfrak{C h} /\{\mathfrak{W}\}=$ $\mathfrak{C h}_{h}$ become strict equivalences (hence isomorphisms) in a universal way.

Finally, we assume that a chain functor $\boldsymbol{C}_{*}$ satisfies:

CH7: All chain complexes $C_{*}(X, U)$ are canonically free (i.e. all $C_{n}(X, U)$ are free abelian groups with a natural basis $\boldsymbol{b}$ (cf. Theorem 3.1 and [4], Lemma 9.1).

\section{Review of simplicial model structures}

Although we assume that the reader is familiar with the definition of a simplicial model structure, it is advisable to note briefly that the two axioms SM6, SM7 of a simplicial model structure must be fulfilled in addition to CM1-CM5. In the text, we will always point out what special modifications are necessary when dealing with the category $\mathfrak{C h}$. Concerning simplicial model categories, we refer the reader to [6] concerning further details or to any other excellent treatment of this subject; e.g. D. Quillen's original survey [11] or M. Hovey's book [8].

Definition 11.1. (SM6) A category $\mathfrak{C}$ is a simplicial category whenever there exists a mapping space functor

$$
\operatorname{Hom}_{\mathfrak{C}}(-,-): \mathfrak{C}^{o p} \times \mathfrak{C} \longrightarrow \mathfrak{S}
$$

such that for objects $A, B \in \mathfrak{C}$

$$
\operatorname{Hom}_{\mathfrak{C}}(A, B)_{0}=\operatorname{hom}_{\mathfrak{C}}(A, B)
$$

(2) the functor

$$
\operatorname{Hom}_{\mathfrak{C}}(A,-): \mathfrak{C} \longrightarrow \mathfrak{S}
$$

has a left adjoint

$$
A \otimes-: \mathfrak{S} \longrightarrow \mathfrak{C}
$$

which is associative in the sense that there is an isomorphism

$$
A \otimes(K \times L) \cong(A \otimes K) \otimes L
$$

which is natural in $A \in \mathfrak{C}$ and in $K, L \in \mathfrak{S}$; 
(3) the functor $\operatorname{Hom}_{\mathfrak{C}}(-, B): \mathfrak{C}^{o p} \longrightarrow \mathfrak{S}$ admits a left adjoint

$$
B^{K}: \mathfrak{S} \longrightarrow \mathfrak{C}^{o p} \text {. }
$$

Some authors call this an enrichment of the category $\mathfrak{C}$. Someone primarily interested in the existence of the tensor product will talk about about tensored categories. There are several other terminologies in use.

Definition 11.2. (SM7) Assume that $\mathfrak{C}$ is a closed model category and a simplicial category in the sense of Definition 11.1. Let $q: A \longrightarrow B$ be a cofibration and $p: X \longrightarrow Y$ be a fibration. Then

$$
\operatorname{Hom}_{\mathfrak{C}}(B, X) \stackrel{\left(j^{*}, p_{*}\right)}{\longrightarrow} \operatorname{Hom}_{\mathfrak{C}}(A, X) \times_{\operatorname{Hom}_{\mathfrak{C}}(A, Y)} \operatorname{Hom}_{\mathfrak{C}}(B, Y)
$$

is a fibration in $\mathfrak{S}$ (hence a Kan fibration) which is a trivial fibration if $j$ or $p$ is trivial.

There is a dual formulation which in a model category turns out to be equivalent to SM7:

Definition 11.2' (SM7) Let $q: \boldsymbol{A}_{*} \subset \boldsymbol{B}_{*}$ in $\mathfrak{C}$ and $j: K \subset L$ in $\mathfrak{S}$ be cofibrations. Then there exists a pushout

$$
\boldsymbol{P}_{*}=\boldsymbol{A}_{*} \otimes L \cup_{\boldsymbol{A}_{*} \otimes K} \boldsymbol{B}_{*} \otimes K
$$

in $\mathfrak{C}$ and a mapping

$$
w=\left(\boldsymbol{B}_{*} \otimes j, q \otimes L\right): \boldsymbol{P}_{*} \longrightarrow \boldsymbol{B}_{*} \otimes L
$$

which is a cofibration. Moreover $w$ is a trivial cofibration whenever $q$ or $j$ is a trivial cofibration.

For the case of the category $\mathfrak{C h}$, we are not able to introduce a tensor product $\otimes$ and a function object $\boldsymbol{A}_{*}^{K}$ satisfying all conditions of Definition 11.1. There are modifications necessary which are explained in detail in $\S 1-\S 4$.

In $\S 1$, a tensor product is established which is not fully associative but only associative up to weak equivalences ( $§ 2$ (3), Lemma 2.7). The existence of $\mathbf{H o m}_{\mathfrak{C h}}=$ Hom is the subject of $\S 4$, Lemma 4.1. In our case, the function object $\boldsymbol{A}_{*}^{K}, K \in$ $\mathfrak{S}, \boldsymbol{A}_{*} \in \mathfrak{C h}$ appears in a larger category $\mathfrak{C h} \subset \mathfrak{C h}$, whose relationship with $\mathfrak{C h}$ is treated in $\S 3.1$ and 3.2. The required adjointness between the tensor product and the function object is displayed in Theorem 4.6.

The two different formulations of SM7 are the subject of $\S 5$ for the tensor product formulation and $\S 6$ for the Hom formulation.

In order to have a notation available, we call this modified simplicial model structure an approximate simplicial model structure and we consequently speak of an approximate simplicial model category.

We can now summarize the results of $\S 1-\S 6$ :

Theorem 11.3. The category $\mathfrak{C h}$ carries the structure of an approximate simplicial model category.

Special remarks concerning the specific situation of the category $\mathfrak{C h}$ are always included. 
Homology, Homotopy and Applications, vol.9(1), 2007

\section{References}

[1] F. W. Bauer, The Boardman category of spectra, chain complexes and (co-)localizations, Homology Homotopy Appl. 1(3) (1999) 95-116.

[2] F. W. Bauer, Tensor products of spectra and localizations, Homology Homotopy Appl. 3(3) (2001) 55-85.

[3] F. W. Bauer, T. Datuashvili, The existence of certain (co-)limits in the category of chain functors, J. Algebra Appl. 5(4) (2006) 379-401.

[4] F. W. Bauer, T. Datuashvili, Model category structures on the category of chain functors, Topology Appl. 131 (2003) 101-128

[5] A. Dold, Lectures on Algebraic Topology, Die Grundlehren d. mathem. Wissenschaften, Springer (1972).

[6] P. G. Goerss and J. F. Jardine, Simplicial Homotopy Theory, Progress in Math. 174, Birkhäuser, Basel-Boston-Berlin (1999).

[7] P. S. Hirschhorn, Model categories and their localizations, AMS Math. Surveys and Monographs 99 (2003).

[8] M. Hovey, Model Categories, AMS Math. Surveys and Monographs 63 (1999).

[9] D. M. Kan, Semisimplicial spectra, Illinois J. Math. 7 (1963) 463-478.

[10] D. M. Kan and G. W. Whitehead, The reduced join of two spectra, Topology 3, suppl. 2 (1963) 239-261.

[11] D. G. Quillen, Homotopical Algebra, Lecture Notes in Mathematics, 43, Springer-Verlag (1967).

Friedrich W. Bauer f.w.bauer@mathematik.uni-frankfurt.de

Fachbereich Mathematik

Johann Wolfgang Goethe Universität

Robert-Mayer Str. 8-10

60054 Frankfurt a.M.

Germany

Tamar Datuashvili tamar@rmi.acnet.ge

A. Razmadze Mathematical Institute

Georgian Academy of Sciences

380093 Tbilisi

Georgia

Alexidze Str. 1

This article is available at http://intlpress.com/HHA/v9/n1/a4 